\title{
A MIXED METHODS STUDY OF COPING AND DEPRESSION IN ADOLESCENT GIRLS WITH POLYCYSTIC OVARY SYNDROME
}

\author{
by \\ CASEY S. HOPKINS \\ A Dissertation Submitted to the Graduate Faculty \\ of Georgia Baptist College of Nursing of Mercer University \\ in Partial Fulfillment of the \\ Requirements for the Degree
}

DOCTOR OF PHILOSOPHY

Atlanta, GA

2016 
ProQuest Number: 10302140

All rights reserved

INFORMATION TO ALL USERS

The quality of this reproduction is dependent upon the quality of the copy submitted.

In the unlikely event that the author did not send a complete manuscript and there are missing pages, these will be noted. Also, if material had to be removed, a note will indicate the deletion.

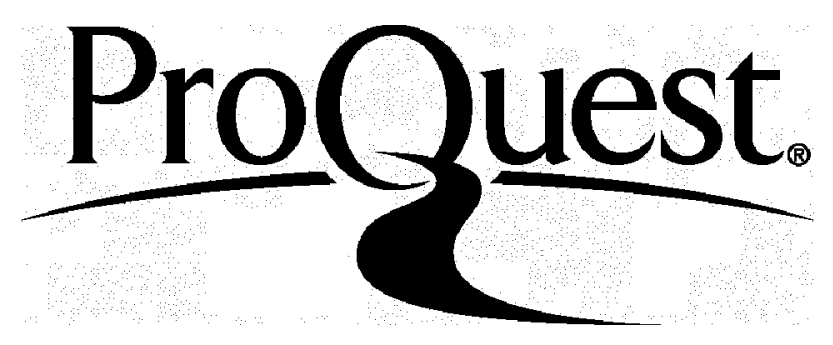

ProQuest 10302140

Published by ProQuest LLC(2017). Copyright of the Dissertation is held by the Author.

All rights reserved.

This work is protected against unauthorized copying under Title 17, United States Code. Microform Edition (C) ProQuest LLC.

ProQuest LLC

789 East Eisenhower Parkway

P.O. Box 1346

Ann Arbor, MI 48106-1346 
(C) 2016

Casey S. Hopkins

All Rights Reserved 


\section{DEDICATION}

I dedicate this dissertation to my husband, Chris, and my daughter, Charleigh. Chris, without your unwavering support completing my $\mathrm{PhD}$ would not have been possible. Charleigh, you were born during the first semester of my doctoral program, and you have filled my days with joy ever since. What a challenging and special journey it has been; I would not have had it any other way. "Now to him who is able to do immeasurably more than all we ask or imagine, according to his power at work within us, to him be the glory in the church and in Christ Jesus throughout all generations, forever and ever. Amen." Ephesians 3: 20-21. 


\section{ACKNOWLEDGMENTS}

I would like to recognize my outstanding dissertation committee for their expertise, guidance, support, and for the time they invested in my dissertation research. I am immensely grateful to you all for mentoring me through this process and for your tremendous contribution to my development as a scholar.

I would also like to thank the deans, faculty, and staff of both Georgia Baptist College of Nursing of Mercer University and the school of nursing of Anderson University for their support during my doctoral program.

I would like to recognize my supervising physician at the healthcare institution where the study took place for her support of my dissertation research. Also, I thank the director of nursing research at the healthcare institution for her guidance through the institutional process for nursing research, and the physicians at the various study sites for identifying eligible participants and allowing me to gain access to their patients.

I thank the study participants for their time and contributions. Their willingness to complete the surveys and participate in interviews was instrumental to the completion of my dissertation research.

Finally, I would like to recognize the funding sources for my doctoral program, the Nurse Faculty Loan Program and the Southern Baptist Foundation Doctoral Loan Program. The financial support I received from these programs made it possible for me to pursue my PhD in Nursing. 


\section{TABLE OF CONTENTS}

Page

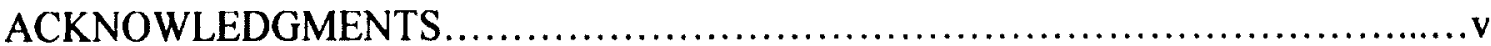

LIST OF TABLES ......................................................................

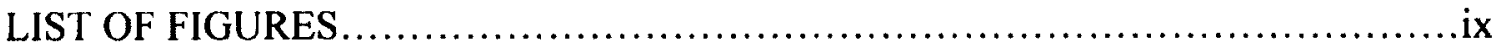

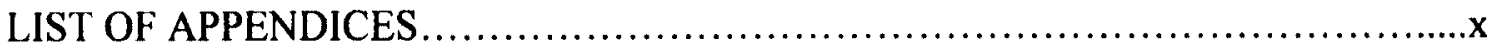

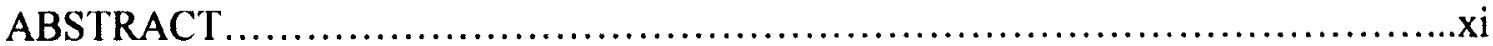

\section{CHAPTER}

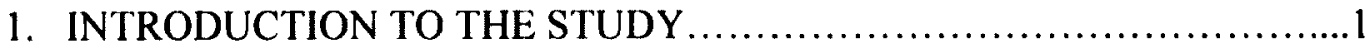

Statement of the Problem.......................................................2

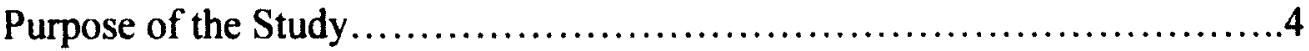

Research Questions.......................................................

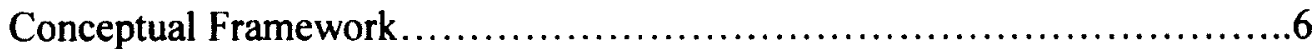

Significance of the Study ............................................ 14

Definition of Terms...................................................... 14

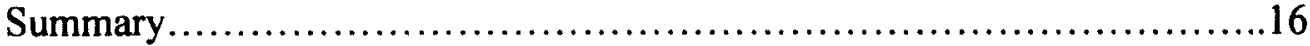

2. LITERATURE REVIEW ..............................................

Diagnosing and Managing PCOS in Adolescents........................17

Psychosocial Dysfunction in Adult Females with PCOS.....................22

Psychosocial Dysfunction in Adolescent Girls with PCOS ...................27

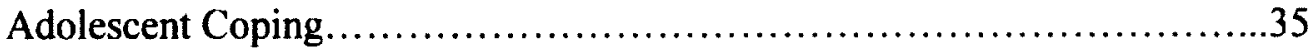

Inferences for Current Study .........................................40

Summary ............................................................... 41

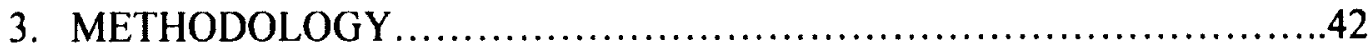

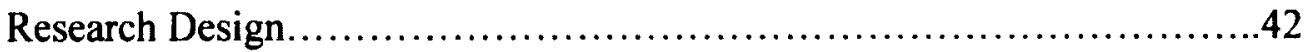

Setting and Sample ........................................................

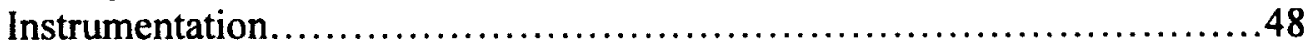

Demographic Characteristics....................................48 
PCOS Stressor Scale ...............................................48

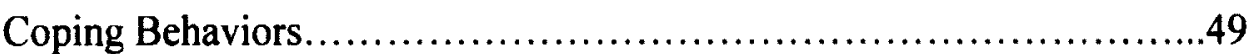

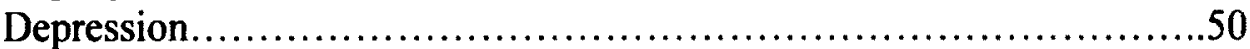

Qualitative Data Collection.......................................52

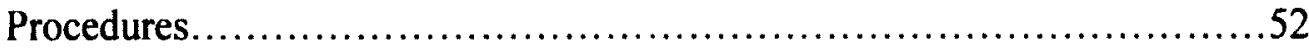

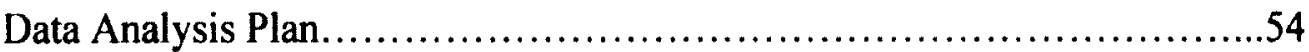

Protection of Human Subjects......................................55

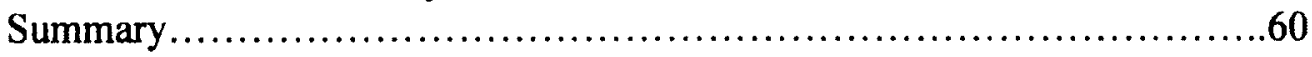

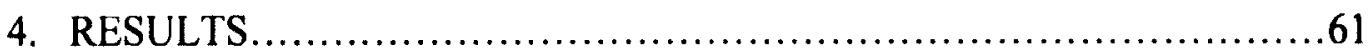

Overview of Data Analysis..............................................61

Description of the Sample.............................................62

Psychometric Properties of Study Instruments...........................66

Depression Among Adolescent Participants...............................67

Data Analysis Addressing Research Questions...........................68

Summary ...............................................................

5. DISCUSSION AND RECOMMENDATIONS ...........................90

Discussion of Findings...........................................99

Girls' Concerns and Perceived Maternal Concerns.......................91

Girls' Perceived Control..................................................99

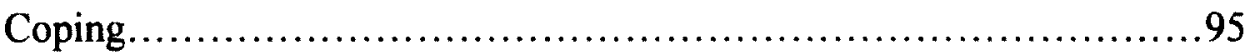

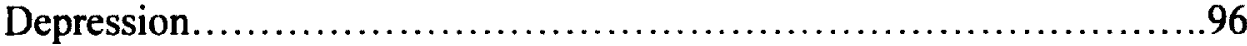

Recommendations for Nursing Practice.............................. 103

Recommendations for Future Research...............................105

Limitations of the Study ...........................................107

Conclusions........................................................ 108

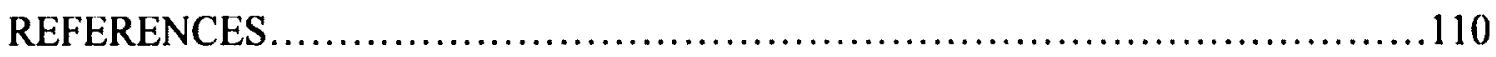

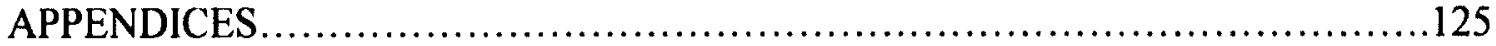




\section{LIST OF TABLES}

Table 1. Demographic Characteristics of Sample

Table 2. Internal Consistency Reliability of Study Instruments

Table 3. Descriptive Statistics for Girls' Concerns about PCOS Stressors

Table 4. Descriptive Statistics for Girls' Control over PCOS Stressors

Table 5. Types and Extent of Coping Behaviors used by Girls with PCOS

Table 6. Descriptive Statistics for Girls' Perceptions of Maternal Concern over PCOS Stressors

Table 7. Pearson Product Moment Correlations between Girls' Perceptions of Maternal Concern and Coping Behaviors

Table 8. Results of Multiple Linear Regression Analysis of Perceived Concern, Perceived Controllability, Types and Extent of Coping Behaviors, and Perceived Maternal Concern as Predictors of Depression in Adolescent Girls with PCOS 


\section{LIST OF FIGURES}

Figure 1. Model of Stress and Appraisal in Adolescent Girls with PCOS

Figure 2. Convergent Parallel Design Diagram for Study 


\section{LIST OF APPENDICES}

Appendix A. Demographic Form

Appendix B. PCOS Stressor Scale

Appendix C. Brief COPE

Appendix D. Patient Health Questionnaire (PHQ-9)

Appendix E. Interview Guide

Appendix F. Institutional Review Board Approval 


\section{ABSTRACT}

\section{CASEY S. HOPKINS}

\section{A MIXED METHODS STUDY OF COPING AND DEPRESSION IN ADOLESCENT GIRLS WITH POLYCYSTIC OVARY SYNDROME Under the direction of Dr. Laura P. Kimble}

Polycystic ovary syndrome (PCOS) is associated with clinical manifestations including obesity, menstrual irregularities, acne, hirsutism, and infertility. The prevalence of psychological disorders among women with PCOS has been well documented in the literature; however, little is known about adolescents' experiences with PCOS. The purpose of this study was to explore relationships among stressors related to PCOS, coping, and depression in adolescent girls with PCOS. Using a convergent parallel mixed methods design, a convenience sample consisting of 23 adolescent girls between the ages of 13 and 18 were recruited from an urban academic healthcare system in the southeastern region of the United States. The measures for this study included demographic questions, two standardized questionnaires to measure coping and depression (Brief COPE and Patient Health Questionnaire 9-item tool), a researcher-developed PCOS Stressor questionnaire (PCOSSS), and a semi-structured interview guide.

Participants reported menstrual irregularities and the threat of future infertility to be the most stressful and least controllable aspects of PCOS. Participants also perceived 
their mothers to be most concerned about menstrual irregularities and future infertility. Acceptance and self-distraction were the coping behaviors most frequently used by the participants. As girls perceived their mothers to be more concerned about aspects of PCOS, the girls' use of acceptance to cope with stress increased. Results indicated perceived control to be a significant predictor of depression; therefore, as girls perceived less control over PCOS-related stressors their depression scores increased, indicating greater depression.

The qualitative data analysis revealed four themes. Three of the themes focused on participants' concerns about PCOS including not understanding the disease process, dealing with uncertainties regarding menstrual irregularities and infertility, and feeling out of control. The last theme revealed the participants' overall experience with PCOS described as not horrible, not the best. The continued study of adolescent girls' experiences with PCOS will allow for the planning of appropriate psychosocial care in order to increase quality of life and decrease the risk of negative psychosocial outcomes for this population. 


\section{CHAPTER ONE \\ INTRODUCTION TO THE STUDY}

Polycystic ovary syndrome (PCOS) is one of the most common endocrine disorders affecting approximately $6-15 \%$ of all reproductive-age women (Youngster et al. 2014). As a syndrome, PCOS is not simply diagnosed clinically by a single criterion; rather the diagnosis is one of exclusion (The Rotterdam PCOS Consensus Group, 2004). According to the Rotterdam PCOS Consensus Group (2004), to establish the diagnosis of PCOS in adult women other disorders must be excluded and two of three following criteria must be present: a) oligo- or anovulation, b) clinical and/or biochemical signs of hyperandrogenism, and c) polycystic ovaries. The diagnosis is difficult to make during adolescence because often the symptoms of PCOS may be dismissed as being part of the normal physiological process of puberty (Agapova, Cameo, Sopher, \& Oberfield, 2014). While there are no universally accepted criteria for the diagnosis of PCOS in the adolescent population, one proposal has been to require all three Rotterdam criteria for diagnosis in an adolescent girl (Hansen, 2014). Others suggest not making a PCOS diagnosis until a woman is at least 18 years old (Agapova et al., 2014). Nevertheless, experts agree clinical signs and symptoms of PCOS should not be ignored in the adolescent population, but should be addressed and appropriately managed due to the potential long-term sequelae of health implications (Anderson, 2006; Collier \& Rieder, 2008; Nicandri \& Hoeger, 2012). 
A hyperandrogenic disorder characterized by clinical manifestations such as menstrual irregularities, hirsutism, alopecia, obesity, acne, and infertility, PCOS is a complex metabolic syndrome which increases an individual's risk for cardiovascular disease and type 2 diabetes (Weiss \& Bulmer, 2011). Considering the clinical expressions of PCOS, a potential negative impact on psychological health could certainly be anticipated. Studies have found women with PCOS have higher rates of eating disorders, fear of social rejection, body dissatisfaction, anxiety, and depression (Dorkas, Clifton, Futterweit, \& Wild, 2011; Karacan, Caglar, Gürsoy, \& Yilmaz, 2014; Trent, Rich, Austin, \& Gordon, 2002). Therefore, when caring for adolescent girls, it is important for health care providers to look beyond the physiological implications of PCOS. Adolescence is a developmental stage in which awareness of oneself and concern regarding body image peaks, thus experiencing and coping with the manifestations of PCOS is a stressful and challenging process for adolescent girls (Collier \& Rieder, 2008). An estimated 60 to 70 percent of adolescents with PCOS are obese and present with hirsutism (Agapova et al., 2014). Considering these appearance-related consequences of the syndrome alone, it is understandable that adolescent girls with PCOS have reported a lower health-related quality of life that their unaffected peers (Jones, Hall, Lashen, Balen, \& Ledger, 2011; Trent et al., 2002).

\section{Statement of the Problem}

Research on the psychosocial aspect of PCOS, in general, has not been addressed adequately in the literature until the past decade (Weiss \& Bulmer, 2011). Considering the prevalence of eating disorders, anxiety, and depression in samples of women with 
PCOS demonstrated by the research and the evident emotional needs and decreased quality of life in teens with PCOS, research is needed in examining the prevalence of psychological disorders, such as depression in adolescents with PCOS (Anderson, 2006; Dorkas et al., 2011; Dowdy, 2012; Kerchner, Lester, Stuart, \& Dorkas, 2009) and the correlates of these disorders. While researchers have demonstrated the psychosocial distress of adolescent girls with PCOS, there is a deficit in the literature addressing the ways in which adolescents cope with the diagnosis of PCOS and all that it entails (Dowdy, 2012). Benson et al. (2010) found passive or maladaptive coping strategies to be strongly correlated with psychiatric symptoms of anxiety and depression in women with PCOS. Further, passive coping strategies predicted compromised quality of life (Benson et al., 2010). Considering adolescent development, the adverse effects of depression are multifaceted, impacting overall well-being, school performance, peer and family relationships, and the risk for dangerous behaviors including substance abuse (Dowdy, 2012). Thus, understanding perceived threats and ways of coping in this population of adolescents is essential in increasing quality of life and decreasing the risk of negative psychosocial outcomes.

Coping has been identified as a key concept for research involving health and adaptation to chronic illness and is defined as "constantly changing cognitive and behavioral efforts to manage specific external and/or internal demands that are appraised as taxing or exceeding the resources of the person" (Lazarus \& Folkman, 1984, p. 178). Perceived control over stressors can impact one's ability to cope (Lazarus \& Folkman, 1984). Compas, Malacarne, and Fondacaro (1988) demonstrated the impact of perceived 
control on the types of coping strategies utilized by adolescents. When the adolescent believed he or she had more control over a certain stressor, generating problem-focused alternatives for coping was associated with less behavioral problems. Therefore, as adolescence is a stage in which teens are learning to take responsibility for their health independent of their parents, the perceptions of control over the stressors associated with PCOS is important in understanding coping among this population (Dowdy, 2012).

On the other hand, while teens are seeking independence and a separate identity from their parents, research has demonstrated the correlation between a mother's body image and the body image reported by her daughter (Izydorczyk, 2010; Usmiani \& Daniluk, 1997); therefore, the mother's perception of the stressful signs and symptoms associated with PCOS may have bearing on the daughter's perception of stressors thus impacting her ability to cope. Based on clinical observations pointing to the presence of this phenomenon and the lack of existing research addressing mother-daughter perceptions of PCOS related to coping the researcher will address this variable in this study. In summary, as poor psychological functioning is related to increased mortality, higher health care costs, and negative health outcomes, and coping has been identified as impacting health and adaptation to chronic illness, greater understanding of the coping process in adolescent girls with PCOS is needed. (Rofey et al., 2009).

Purpose of the Study

Therefore, the purpose of this study was to explore relationships among PCOSrelated stressors, coping, and depression among adolescent girls with PCOS. A convergent parallel mixed methods design was used to quantitatively examine coping and 
depression while simultaneously using semi-structured interviews to qualitatively explore the participants' experiences with PCOS. The quantitative and qualitative data will be merged to provide insight into explaining perceptions of stress and control, coping processes, and depression scores among adolescent girls with PCOS.

Research Questions

The study addressed the following research questions:

Quantitative Research Questions:

1. What aspects of PCOS do adolescent girls with PCOS perceive as most stressful?

2. What aspects of PCOS do adolescent girls with PCOS perceive as most controllable?

3. What types and extent of coping behaviors do adolescent girls use to cope with PCOS?

4. Are adolescent girls' perceptions of their mothers' feelings regarding aspects of PCOS associated with type and extent of coping behaviors used by adolescent girls with PCOS?

5. Are perceived stressors, perceived controllability, types and extent of coping behaviors, and adolescent girls' perceptions of their mothers' feelings regarding aspects of PCOS predictors of depression in adolescent girls with PCOS?

Qualitative Research Question:

6. What are the experiences of adolescent girls in coping with the stressors associated with PCOS? 
Mixed Methods Research Question:

7. To what extent do the quantitative results about stressors, perceived control, coping, and depression converge with the interview data reporting the coping experiences of adolescent girls with PCOS?

\section{Conceptual Framework}

Understanding how one copes with stressful events and being able to recognize the effects of coping on one's overall psychological health is an integral component in promoting health and wellness during transitional times (McHaffie, 1992). Studying the concept of coping in individuals not only explains variability in stress responses, but leads to an understanding of appropriate cognitive-behavioral interventions to improve overall health (Folkman \& Moskowitz, 2004). Lazarus' theory of stress and coping served as the conceptual framework for this current study regarding coping in adolescent girls with PCOS.

A basic assumption of Lazarus' theory of stress and coping is the existence of individual differences in evaluating stressful events (Lazarus \& Folkman, 1984). Lazarus and Folkman (1984) defined psychological stress as a "particular relationship between the person and the environment that is appraised by the person as taxing or exceeding his or her resources and endangering his or her well-being" (p. 19). Stress may present as a harm, threat, or challenge. The way stress is interpreted or appraised by an individual results in emotional or behavioral coping responses. In view of that, an assumption of the theory is viewing stress as a transaction between an individual and his or her environment (Krohne, 2001). The process of cognitive appraisal encompasses the dynamic 
relationship between a person having unique past experiences which have influenced a set of values, beliefs, and perceptions and the surrounding environment in which events must be anticipated and interpreted. Therefore, any given event or circumstance is not inherently bad or good, but how one construes the situation determines the characteristics of the event and thus determines one's coping processes and outcomes (Lazarus \& Folkman, 1984).

Lazarus and Folkman (1984) established two distinct forms of appraisal: primary appraisal and secondary appraisal. It is important to understand that neither one form of appraisal is more favorable than the other, nor does one form of appraisal necessarily precede the other in any given circumstance. Primary appraisal is concerned with determining the presence of stress, currently or in the future, and what form the stress is taking. According to Lazarus (1999) stress and emotion are interdependent, therefore, whether the stressful event is interpreted as positive, neutral, or negative determines the resulting emotion. Stressors perceived as challenges are associated with positive emotions whereas negative emotions are typically expected in cases of threats and/or harm. Chronic diseases are usually appraised as threats to the physical or psychological self therefore typical emotional responses are fear, anxiety, depression, anger, grief (Maes, Leventhal, \& De Ridder, 1996). In the case of adolescent girls faced with the diagnosis of PCOS, negative emotional responses would be expected. However, according Lazarus' theory of stress and coping, over time the immediate responses to the diagnosis would decrease but specific stressors associated with the syndrome may continue to pose threats. For example, upon diagnosis the adolescent may be anxious 
about the uncertainty surrounding long-term effects of PCOS including the increased risk for chronic diseases or infertility and may grieve the loss of what she perceived as normality; however, as times passes those feelings may decrease and certain situations such as dealing with the maintenance of facial hair or menstrual irregularities may become a more regular source of anxiety (Maes et al., 1996).

Whereas primary appraisal takes into account the existence and nature of stress, secondary appraisal involves examining what can be done about the stressor in order to decrease the threat, loss, or harm inflicted by the stressor (Lazarus \& Folkman, 1984). During this type of appraisal one evaluates the available coping processes and determines the effectiveness of those processes. Together primary and secondary appraisals interact to shape the overall magnitude and significance of the stressor. For example, if an adolescent girl interprets being obese as harmful to her self-esteem and body image and feels helpless and unable to lose weight then the stress will be overwhelming because of the girl's inability to lessen or overcome the threat, loss, or harm. These thoughts of action involved in the secondary appraisal process are referred to as coping capacities (Maes et al., 1996). Lazarus and Folkman (1984) describe coping as a dynamic, processoriented approach to managing psychological stress which is appraised to be taxing or exceeds the resources one has available to manage the internal or external demand.

Considering an individual's coping capacity, Lazarus and Folkman (1984) described two coping processes including problem-focused and emotion-focused coping. The two main functions of coping are to manage, which could mean to minimize, tolerate, avoid, or accept, the stressor and to regulate the emotional response incurred by 
the stressor. Problem-focused responses are aimed at the external situation causing the stress and emotion-focused coping is directed at the individual's internal state resulting from the external stress. An example of problem-focused coping may be to develop new behaviors, learn new skills, or alter one's environmental barriers or resources (Lazarus \& Folkman, 1984). An example of a problem-focused coping behavior in adolescent girls dealing with hirsutism associated with PCOS would be scheduling appointments for laser hair removal or waxing, whereas, an example of emotion-focused coping would include responses to stressors including distancing one's self from a stressful situation, denial, avoidance, making positive comparisons, etc. Although maladaptive, an example of an emotion-focused coping behavior in an adolescent girl experiencing depression and anxiety related to the stress of symptoms associated with PCOS may be the use of alcohol or drugs to lessen the negative emotions she is feeling. Problem and emotion-focused coping strategies may occur simultaneously and may facilitate or create barriers for certain ways of coping to take place (Lazarus \& Folkman, 1984). Furthermore, problem and emotion-focused ways of coping are not mutually exclusive (Lazarus, 1996). Many coping behaviors serve to both address the problem at hand and the emotional response. For instance, planning in response to a stressor may guide problem-solving strategies while calming emotions or provoking greater anxiety. Therefore, when considering coping behaviors used by adolescent girls with PCOS, it is important to understand that the type of behavior used is not a singular function, but is a behavior type with many functions (Lazarus, 2006). 
Regardless of the type of process, an important concept of Lazarus' coping theory is that coping is indeed a process rather than a static change. Processes are described as fluid changes occurring in the individual over time (Lazarus, 1993). In the same way, appraisals of stress are also a process which is subject to change over time considering the context of the stressor. Considering the appraisal of stress, the context of stress, and the shape the stress takes on as the encounter unfolds, coping is shifting process requiring an individual to employ varied coping responses and behaviors depending on the situation at hand (Lazarus \& Folkman, 1984).

Control is an important variable in coping with stress. Lazarus and Folkman (1984) noted the rarity of finding discussion surrounding stress, appraisal, and coping without addressing the issue of personal control. Utilized by different authors in varied contextual meanings, control is not defined as a specific concept, but rather has multiple meanings. Control can be viewed as an individual characteristic or antecedent to a stressor, as a coping process, or as an outcome (Lazarus \& Folkman, 1984). When regarding control as an effort to manage a stressful encounter, control and coping become synonymous. Thus, for the purposes of this study, when control implies effort the term coping will be utilized to describe the response, and the term control will be used solely to describe beliefs influencing emotions or behaviors.

As a belief influencing on one's appraisal of stress, control involves feelings of confidence and mastery. Control as an antecedent may be considered generalized or situation-specific, whereas generalized control is seen as a stable disposition and situation-specific control has to do with one's evaluation of the situation and the resulting 
perceived influence over that situation considering available coping resources and ability to implement coping strategies. Most research has supported a positive correlation between perceived control and decreased stress (Lazarus \& Folkman, 1984).

Nevertheless, there are circumstances in which control may instigate negative coping responses especially when control is exerted over certain personal values or beliefs causing internal conflict and moral distress. Considering the purpose of this study in evaluating the relationship between depression and coping, it is important to note the correlation between depression and a general expectancy of little or no control. Additionally, depressed persons more commonly apply emotion-focused coping strategies rather than problem-focused strategies when the stressor is appraised as changeable, meaning some degree of control is perceived (Zeidner \& Saklofske, 1996). Interestingly, Compas et al. (1988) found emotional and behavioral problems were more likely to occur in adolescents utilizing emotion-focused coping to deal with stress perceived as controllable than those matching the controllable circumstance with problem-focused alternatives. Regardless of whether beliefs of control are general, specific, realistic, or illusory, the beliefs do affect one's appraisal of stress and the outcomes of the coping process (Lazarus \& Folkman, 1984).

Finally, how one appraises and copes with stress may result in three major classes of outcomes outlined by Lazarus and Folkman (1984) including social functioning, morale, and somatic illness. Consequences of coping do not result in one certain adaptational outcome, but rather a dynamic interplay of each outcome. First, social functioning is defined as the "ways the individual fulfills his or her various roles, as 
satisfaction with interpersonal relationships, or in terms of the skills necessary for maintaining roles and relationships" (Lazarus \& Folkman, 1984, p. 223). How an individual functions socially is determined by the effectiveness and appropriateness of appraisal and coping considering the context of the stressful event. In the long-term, morale has to do with the one's ability to appraise circumstances as challenges and manage negative events with a positive outlook. Therefore, one's collective emotions and acceptance of circumstantial outcomes over time influence morale. For example, if an individual learns to cope by acting and feeling helpless, a state of depression often results. This is an example of damaged morale as an adaptational outcome. Lastly, somatic illness may result when there is failure to regulate emotional distress, use of harmful substances as coping mechanisms, and/or the adoption of other self-destructive behaviors including denial or avoidance. The relationships between these three adaptational outcomes are complex and the impact of coping with stress can certainly affect an individual's overall health including both the physical and psychological aspects of well-being (Lazarus \& Folkman, 1984).

Lazarus' and Folkman's (1984) model of stress and coping is useful in studying coping among adolescents. Compas et al. (1988) found older children and young adolescents were able to utilize both problem-focused and emotion-focused coping strategies in response to perceived stressors. Therefore, in evaluating ways of coping in adolescent girls with PCOS the basic assumptions and concepts described in Lazarus' theory of coping informed the conceptualization and measurement of variables in this study. Informed by Lazarus' and Folkman's (1984) model of stress and coping, Figure 1 
depicts the process of coping as experienced by adolescent girls confronted with the threats and challenges of PCOS.

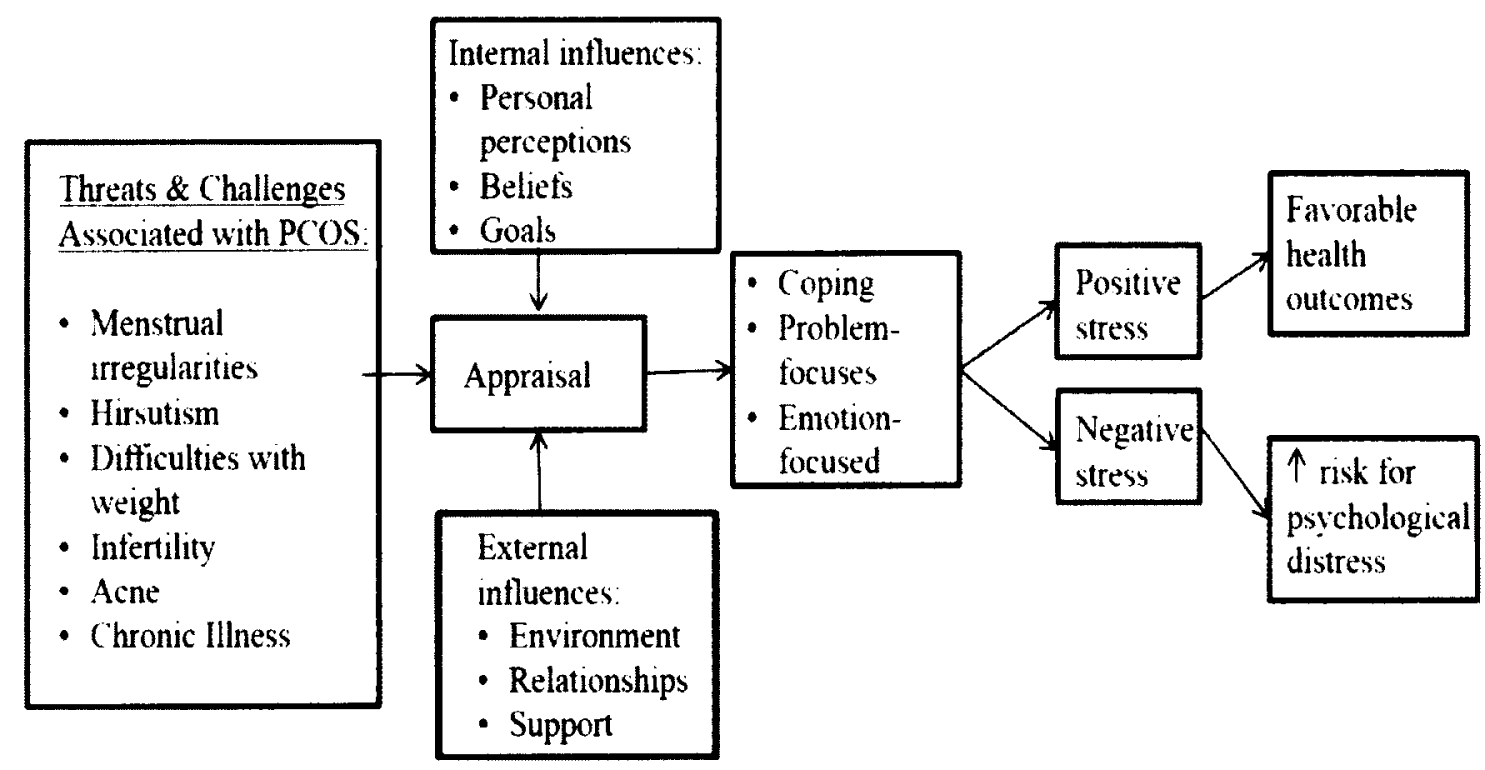

Figure 1. Model of Stress and Appraisal in Adolescent Girls with Polycystic Ovary Syndrome. Adapted from Lazarus \& Folkman, 1984.

Significance of the Study

This study adds significantly to the body of knowledge surrounding PCOS by identifying relationships between perceived stress, control, coping strategies and depression scores among adolescent girls with PCOS. Furthermore, the study represents 
the first step toward discovering the impact mother-daughter perceptions have on coping in adolescent girls with PCOS. Finally, as coping behaviors directly influence psychological function, which if negative, can lead to increased mortality and negative health outcomes, the findings from this study are invaluable in planning appropriate psychosocial care for this population (Rofey et al., 2009).

\section{Definition of Terms}

The definitions of terms for the study include:

Polycystic ovary syndrome (PCOS). This term was defined as a complex metabolic syndrome characterized by menstrual irregularity, polycystic ovarian morphology, and hyperandrogenism. Clinical features of hyperandrogenism may include acne, hirsutism, or alopecia. Additionally, PCOS is associated with infertility, obesity, insulin resistance, and increased risk of cardiovascular disease and type II diabetes (Agapova et al., 2014).

Adolescent girls, Girls, Teens, Adolescents. For the purposes of this study, these terms were be used interchangeably to describe the population of interest. The adolescent years begin with the onset of puberty and continue over a span of approximately ten years. Adolescence is often divided into three stages: early (ages 11 to 13), middle (ages 14-17), and late (ages 18-21). The adolescent girls referred to in this study were in the age range from 13 to 18 years. Additionally, for the context of this study, adolescents should be viewed as individuals undergoing immense physical and psychosocial development and change (Schuiling \& Low, 2006). 
Coping. Coping, as it relates to health care, was defined as the ability to actively adapt cognitive and behavioral efforts to manage situations that may be externally or internally imposed which are perceived to exceed one's resources or to be particularly demanding (Lazarus \& Folkman, 1984). Coping as it relates to adolescent girls with PCOS involves the cognitive or behavioral strategies utilized to manage the implications of the syndrome, physically and psychosocially.

Signs and symptoms of PCOS. For the purposes of this study, signs and symptoms of PCOS were considered to be the documented, clinical manifestations of PCOS that individuals may or may not present with. The signs and symptoms may include menstrual irregularity, hirsutism, acne, infertility, overweight, or obesity (Anderson, 2006). An individual may or may not perceive all, none, one, or any combination of these clinical signs and symptoms to be stressful.

Stressors. The stressors associated with PCOS may be the consistent with the signs and symptoms of PCOS; however, stressors can also include long-term health risks related to PCOS such as the threat of chronic disease as well as psychosocial concerns. For example, a girl with PCOS suffering from acne and hirsutism may experience low self-esteem and may have trouble relating to her peers and developing a strong social support system. If an obese girl with PCOS who, despite her efforts, has been unsuccessful in losing weight, has a mother who highly values thinness and does not understand her daughter's inability to lose weight may experience a feelings of hopelessness or rejection. Therefore, stress linked to PCOS could involve an array of physical or psychological causes. 
Sequelae. The term sequelae was defined as "an aftereffect of a disease, condition, or injury" and as "a secondary result" (Sequela, 2015, para 1). In relationship to PCOS, the sequelae refers to the negative long-term health risks associated with the syndrome. The sequelae of health implications associated with PCOS include but are not limited to, metabolic syndrome, cardiovascular disease, type Il diabetes, endometrial carcinoma, and psychological threats such as depression, anxiety and even suicide (Anderson, 2006; Teede et al., 2010).

\section{Summary}

This chapter has provided an overview of the psychological implications of polycystic ovary syndrome on adolescent girls and has identified a gap in the literature surrounding the understanding of ways in which girls cope with the stressors associated with PCOS and certain variables impacting their ability to cope including motherdaughter perceptions and the perception of control. The purpose of this study was to examine coping and depression in adolescent girls with PCOS considering the impact of perceived control and the girls' interpretations of their mothers' perceptions of PCOS. Additionally, the research questions, a detailed discussion of the conceptual framework for the study, the significance of the study, and the definitions of terms were presented in chapter one. 


\section{CHAPTER 2}

\section{LITERATURE REVIEW}

This chapter will provide an evaluation and discussion of the current state of science relevant to coping and depression in adolescent girls with polycystic ovary syndrome (PCOS). The main topics included in this review are: (a) current practice in diagnosing and managing PCOS, (b) psychosocial dysfunction in adult females with PCOS, (c) psychosocial dysfunction in adolescent girls with PCOS and obesity, which is a prominent feature of PCOS, (d) adolescent coping, and (e) mother-daughter relationships and their influence on body image and self-esteem. This chapter will provide evidence in support of a mixed methods study addressing coping and depression among adolescent girls with polycystic ovary syndrome. The chapter will end with inferences for the current study.

Synthesis of Literature

Diagnosing and Managing PCOS in Adolescents

Despite the challenges of diagnosing adolescents with PCOS, the need to identify individuals with these characteristics is imperative considering the long-term health consequences associated with PCOS (Agapova et al., 2014). Utilizing the Rotterdam diagnostic criteria, most experts suggest a conservative approach to management of PCOS in adolescents due to the overlap between physiologic puberty and clinical features of PCOS (Agapova et al., 2014). Collier and Rieder (2008) highlighted common clinical 
and biochemical presentations in girls with PCOS. First, clinically, anovulatory menstrual cycles in adolescent girls may present as dysfunctional uterine bleeding, primary or secondary amenorrhea, or oligomenorrhea (Collier \& Rieder, 2008).

Dysfunctional uterine bleeding is defined as irregular bleeding of endometrial origin which may be prolonged or excessive and is not related to pregnancy, pelvic, or systemic disease (Faucher \& Schuiling, 2006). Primary amenorrhea is the absence of menses by age 14 without development of secondary sexual characteristics or the absence of menses by age 16 without delay in development of secondary sexual characteristics (Faucher \& Schuiling, 2006). Secondary amenorrhea may be diagnosed when menses cease to occur for six months or greater in a previously menstruating female. Finally, oligomenorrhea is defined as a decreased menstrual flow at intervals greater than 35 days apart (Faucher $\&$ Schuiling, 2006). Most commonly, girls with PCOS present with oligomenorrhea (Collier \& Rieder, 2008).

Other clinical signs of PCOS result from hyperandrogenism including hirsutism and acne. Hirsutism may be assessed and quantified by observing sensitive anatomical sites for male pattern hair growth including the upper lip, chin, chest, upper arms, thighs, abdomen, and the upper and lower back (Ferriman \& Gallwey, 1961). Obesity is also an important clinical sign as more than two-thirds of adolescents with PCOS are obese (Agapova et al., 2014). Collier and Rieder (2014) recommended assessing the degree to which an adolescent girl is overweight with indicators of hyperinsulinemia as increased visceral adipose tissue is associated with hyperandrogenism and hyperinsulinemia. Overall degree of obesity should be assessed with a measurement of height and weight to 
calculate the body mass index and visceral obesity should be assessed by measuring waist and hip circumference in order to calculate the waist-to-hip ratio (Collier \& Rieder, 2008). Lastly, a common clinical finding on obese females with PCOS is acanthosis nigricans which is a cutaneous marker of hyperinsulinemia resulting in a darkened, velvety area on the skin. Typically found in skin folds, acanthosis nigricans is most often noted on the nape of the neck, axilla, groin, and vulva (Romo \& Benavides, 2008).

Assessing biochemical features associated with PCOS are important in order to rule out other conditions presenting with menstrual irregularities and/or hyperandrogenism. Laboratory tests commonly ordered to evaluate an adolescent presenting with the clinical signs and symptoms described above include the following: prolactin, serum $\beta$ hCG, 17-hydroxyprogesterone (17-OHP), dehydroepiandrosterone sulfate (DHEAS), thyroid function tests, free and total testosterone, androstenedione, sex hormone binding globulin (SHBG), luteinizing hormone, and follicle stimulating hormone (Collier \& Rieder, 2008; Nicandri \& Hoeger, 2012). Glucose tests are often evaluated in obese individuals to determine the presence of insulin resistance or metabolic syndrome (Collier \& Rieder, 2008). While androgen excess and insulin resistance may provide biochemical evidence of PCOS, normal laboratory values do not exclude the diagnosis of PCOS (Collier \& Rieder, 2008; Nicandri \& Hoeger, 2012).

Finally, a clinical feature of PCOS is the presence of polycystic ovaries on ultrasound. Polycystic ovarian morphology is defined in the Rotterdam diagnostic criteria as the presence of 12 or more follicles in each ovary, measuring 2 to $9 \mathrm{~mm}$ and/or increased ovarian volume (The Rotterdam PCOS Consensus Group, 2004). 
While transvaginal ultrasound is a sensitive and specific tool for determining the existence of ovarian follicles, the test is not often used in the adolescent populations as many of the girls are virginal making the use of the transvaginal probe inappropriate. Therefore, transabdominal ultrasound may be used in adolescents to evaluate for polycystic ovarian morphology. This tool is not commonly used to diagnose adolescents with PCOS for several reasons. First, the transabdominal ultrasound is less discriminating than the transvaginal ultrasound, especially in obese girls where excess adipose tissue limits the usefulness of the imaging. Second, polycystic ovaries are often found in healthy, normal adolescent females; therefore, the presence of polycystic ovaries alone is certainly not enough to invoke suspicion of PCOS (Agapova et al., 2014).

Agapova et al. (2014), Collier and Rieder (2008), and Nicandri and Hoeger (2012) outlined treatment options for adolescents with PCOS. The purpose of treatment is aimed at regulating menses, improving metabolic status, and decreasing androgenic manifestations clinically and biochemically. Since the majority of adolescent girls with PCOS are obese, the first option for therapy is weight loss achieved through lifestyle changes. Kiddy et al. (1992) found women with PCOS experienced improvement in menstrual function and a reduction in hirsutism after losing as little as five percent of their body weight after approximately six months of dietary changes. Weight loss achieved by long-term calorie restriction results in decreased insulin levels and can therefore reduce the risk of chronic health problems including diabetes and cardiovascular disease (Kiddy et al., 1992). Healthcare providers have an important role 
in teaching girls about healthy diets and exercise and encouraging long-term commitment to the goal of weight loss (Nicandri \& Hoeger, 2012).

Other commonly used treatments modalities for PCOS include the use of combined oral contraceptive pills or regular progestin exposure in order to regulate menses, prevent endometrial hyperplasia, and decrease androgen production, therefore, decreasing the risk of endometrial cancer and reducing hirsutism (Collier \& Rieder, 2008; Nicandri \& Hoeger, 2012). Additionally, the use of metformin, and insulin-sensitizing agent, in adolescents with PCOS has been shown to normalize hyperandrogenemia and improve regulation of the menstrual cycle. Eisenhardt et al. (2006) conducted a randomized, double-blind, placebo-controlled trial evaluating the early effects of metformin in women with PCOS. Eighty percent of the participants experienced improvement in menstrual cycles after receiving metformin therapy for 12 weeks in contrast to only 18 percent of the women in the placebo group reporting improvement in menstruation. Ibanez, Valls, Potau, Marcos, and de Zegher (2000) studied adolescent girls using metformin after precocious puberty. After six months of therapy, Ibanez et al. (2000) found a reduction in hirsutism, insulin resistance, circulating androgen levels, and total and low density lipoprotein cholesterol levels. The girls also reported regular menses after four months of metformin therapy. Therefore, the use of metformin is beneficial for adolescents whose symptoms are not controlled by weight loss and the use of combined contraceptive pills alone. Additionally, metformin is effective in decreasing dyslipidemia and hyperinsulinemia which are two major risk factors diabetes and cardiovascular disease (Collier \& Rieder, 2008). 
Psychosocial Dysfunction in Adult Females with PCOS

Unique to the treatment of adolescent girls with PCOS, psychosocial issues emerging during adolescence concerning body image are important to address as the way girls respond to the stressors associated with PCOS may shape the girl's self-perceptions and relationships with others into adulthood (Nicandri \& Hoeger, 2012). Literature surrounding psychosocial dysfunction in adolescents with PCOS is limited; however, research in the adult PCOS population is well documented.

In a study of 95 women with PCOS, Hollinrake, Abreu, Sparks, Van Voorhis, and Dorkas (2007) reported an increased risk of depression in this population to be associated with high body mass index (BMI) and insulin resistance. The investigators noted factors known to influence depression scores such as age, infertility, marital status, and a family history of depression did not differ between the PCOS groups with and without depression. However, the PCOS group with depression was found to have higher fasting glucose levels and higher BMIs. Therefore, the researchers called for further investigation into the effects of weight loss and increased insulin sensitization on depression scores in women with PCOS. Further, after completing a longitudinal study over 26 months, Kerchner et al. (2009) concluded women with PCOS are at a significant risk for mood disorders. The prevalence of depression among participants was 40 percent. From year one to year two, 11 new cases of depression were reported. The majority of the women in the study suffered from a mood disorder. In addition to depression, anxiety and binge eating disorders were prevalent. Similar to the report from Hollinrake et al. (2007), the presence of fertility concerns or issues was not significantly 
different in women with or without depression. However, in contrast to the findings reported by Hollinrake et al. (2007), issues surrounding body image including weight, hirsutism, and acne were not significantly different in women with or without depression. Nevertheless, the most prevalent concerns among participants were related to excess weight (Kerchner et al., 2009). Kerchner et al. (2009) did note anxiety scores could have been related to body image perceptions; however, because they did not determine the severity of hirsutism for each participant, they were unable to calculate correlations between hirsutism and anxiety. Clayton, Lipton, Elford, Rustin, and Sherr (2005) described the benefits of laser hair removal in women with PCOS, including a reduction in depression and anxiety, since most hirsute women spend a great deal of time concealing abnormal hair growth. Ultimately, Kerchner et al. (2009) recommended all women with PCOS be initially and routinely evaluated for mental health disorders such as depression, anxiety, and eating disorders as these conditions increase one's risk for comorbid chronic diseases, disability, and diminished productivity in general.

Over the past several years, researchers have speculated regarding the cause of depression and other mood disorders among women with PCOS. In attempt to explain the prevalence of psychological dysfunction in this population, studies have been conducted to determine the effects of biochemical factors. Weber, Lewicka, Deuschle, Colla, and Heuser (2000) reported a link between depression and high testosterone levels resulting from excess adrenal androgen production. Other studies have reported no difference in the testosterone levels and mood disorders in females (Schmidt, Murphy, Haq, Danaceau, \& Simpson St Clair, 2002). Other investigators have found higher levels 
of free testosterone or treatment with testosterone therapy to be associated with decreased negative moods and a reduction in depressive symptoms (Davis \& Tran, 2001; Weiner, Primeau, \& Ehrmann, 2004). Therefore, the research surrounding a biochemical explanation for mood disorders, namely depression, in women with PCOS is inconclusive. As the hypotheses for biochemical causes continue to contradict each other, the psychosocial variables associated with PCOS have generated interest among researchers.

Himelein and Thatcher (2006) collected data comparing 40 women with PCOS with or without infertility, 40 women with only infertility, and 60 women without PCOS or infertility. Surveys measuring depression scores, body mass index, and body image indicated women with PCOS had more depressive symptoms, higher BMI, and more body dissatisfaction than the other two groups of women. Being overweight or obese was found to contribute to negative moods among women with PCOS, but body dissatisfaction was not related only to weight concerns. In fact, women with PCOS were more concerned with their skin, facial hair, general hair features, and overall appearance. Thus, Himelein and Thatcher concluded appearance-related concerns are of great importance when managing symptoms of PCOS. Overall, body dissatisfaction and depressive symptoms were associated for all women involved in the study; therefore, since women with PCOS are more likely to be faced with factors leading to appearancerelated concerns and body dissatisfaction, they are at higher risk for depressive symptoms and should be counseled and supported by healthcare providers. Based on their findings, Himelein and Thatcher proposed improved body image to be a more important focus in 
the treatment of women with PCOS than weight loss, as failed dieting attempts and weight cycling may only increase the risk for psychosocial dysfunction. Finally, the authors urged healthcare providers to be aware of the complex relationship between dieting, depression, and body dissatisfaction and to provide long-term encouragement and assistance to patients when managing PCOS (Himelein \& Thatcher, 2006).

In order to determine the prevalence of depression among women with PCOS, Dorkas et al. (2011) completed a meta-analysis of published literature from 1950 through 2009. Studies involving cross-sectional comparisons of women with and without PCOS were included in the systematic review. The studies included reproductive age women, with the majority ranging from 20 to 35 years of age. Dorkas et al. reported women with PCOS were four times more likely to have abnormal depression scores than women in the control groups independent of weight. Therefore, despite obesity being linked as a risk for depression, having PCOS may be considered a risk for depression without weight concerns as a contributing factor. In addition to depression, the prevalence of anxiety and eating disorders were also increased in the population of women with PCOS. Suicide attempts were also found to be more likely in women with PCOS (Dorkas et al., 2011).

Dorkas et al. (2011) asserted health-related quality of life is negatively affected by the common clinical manifestations of PCOS resulting in a higher prevalence of body and sexual dissatisfaction, social phobias, body pain and decreased physical, social, and emotional role functioning. Furthermore, major depression has been linked to a higher rate of cardiovascular incidents in both healthy individuals and those with coronary artery disease (Dorkas et al., 2011). Studies have confirmed the risk of cardiovascular disease 
in women with PCOS as they are predisposed to metabolic syndrome and often have traditional risk factors including obesity, dyslipidemia, hypertension, and hyperinsulinemia (Dorkas et al., 2005; Wild, Painter, Coulson, Carruth, \& Ranney, 1985). Given the previously increased risk for cardiovascular disease, compounding the risk of major depression certainly adds to the danger of cardiac events in women with PCOS. Dorkas et al. (2011) concluded their meta-analysis emphasizing the importance of conducting comprehensive evaluations for mood disorders in women presenting with symptoms of PCOS. Early diagnosis and appropriate treatment of mood disorders in this population has the potential to positively impact overall health-related quality of life and decrease the risk of serious cardiac events or suicide (Dorkas et al., 2011).

Regarding coping in the adult population with PCOS, Benson et al. (2010) conducted a study measuring coping, anxiety, depression, and quality of life in women with a mean age of 29 years. This was the first study analyzing coping as a variable in women with PCOS despite evidence documenting apparent threats and challenges faced by women with PCOS including infertility, health risks, and changes in physical appearance. Guided by Lazarus and Folkman's (1984) theory of stress and coping, Benson et al. (2010) recognized coping mechanisms to be an important mediator between the insult or stressful situation and the resulting psychological stress experienced by an individual. Active and passive coping strategies were analyzed and their associations with quality of life and mood disorders including anxiety and depression were assessed. Benson et al. found women with PCOS were more likely to use passive coping strategies which are generally associated with increased stress and symptom severity. In support of 
this assumption, the researchers reported passive coping to be positively related to greater depression and anxiety and lower quality of life. The use of active coping strategies was not found to be related to increased anxiety or decreased quality of life. Depression was only marginally associated with active coping strategies in this study. Therefore, when women are faced with a PCOS diagnosis, understanding the coping strategies they employ may be useful in predicting quality of life and preventing psychological distress resulting in anxiety or depression. Thus, Benson et al. (2010) stressed the importance of assessing coping strategies to determine risks for impaired psychosocial well-being and to implement interventions aimed to assist women in coping more effectively with the implications of PCOS.

Psychosocial Dysfunction in Adolescent Girls with PCOS

While several quantitative studies have been conducted measuring psychosocial dysfunction in adult women with PCOS, very few similar studies exist evaluating the adolescent population. Ghazeeri, Fakih, Abbas, Harajly, and Awwad (2013) and Karacan et al. (2014) conducted quantitative studies involving adolescent females with PCOS in the Middle East. Ghazeeri et al. (2013) sought to assess the prevalence of anxiety and depressive symptoms among adolescents with PCOS. The girls ranged from age 14 to 18 years. Interestingly, the researchers discovered no association between mood disorders and PCOS. This finding was surprising to the researchers given the extensive documentation of correlations between psychosocial dysfunction and PCOS in adult women. Ghazeeri et al. noted that the vast majority of studies examining women with PCOS indicating an association with mood disorders were conducted on women in 
Western populations. Considering their study included only Lebanese adolescents, the researchers expressed interest in examining geographical and cultural perceptions of PCOS among Lebanese women compared to those in Western societies. Despite there being no correlation between mood disorders and PCOS, adolescent girls did report difficulties with being overweight as the most distressing symptoms of PCOS (Ghazeeri et al., 2013).

In a study of Turkish adolescents and young women ranging from 15 to 24 years of age, Karacan et al. (2014) examined the associations between having PCOS and body dissatisfaction and eating attitudes. All participants in the study were within a health range for body weight as measured by having a $\mathrm{BMI}$ below 25 in order to control for obesity as a confounding variable. Karacan et al. found body esteem to be an important predictor of eating attitudes in both the control group and the PCOS group. Both groups of girls were found to idealize thinness and generally considered their bodies larger than the ideal body size or shape. However, the PCOS group did have a slightly higher tendency to internalize the thin Western ideal body type which negatively impacted body satisfaction and was associated with eating attitudes. Upon observing for differences between the adolescent girls and young women in the study, Karacan et al. discovered the young women over the age of 19 had significantly higher body appearance esteem than the adolescent girls.

While this study only included girls at a normal weight, it is important to recall the majority of girls with PCOS are obese; therefore, body dissatisfaction is certainly an area of concern given the impact of body dissatisfaction and risk of eating disorders and 
mood disorders. In a study conducted by Adali et al. (2008), women with PCOS were found to have significantly higher levels of depression and emotional distress than their healthy counterparts. The prevalence of depression and emotional distress was even more significant among the obese women with PCOS included in the study.

Nevertheless, Karacan et al. (2014) determined sociocultural body ideals to equally affect the body satisfaction and eating attitudes of adolescent girls and young women regardless of PCOS symptoms.

Based on an integrated review of the literature, Dowdy (2012) reported the primary concerns of teens with PCOS and the emotional needs they possess. Dowdy found teens often desire more information about having PCOS, the implications of the syndrome, and how to best manage the symptoms. Dowdy generated a list of emotional needs of teens based on her review of literature and popular internet-based support sites for teens with PCOS. Teens wanted to feel supported by others going through similar hardships and were seeking ways to improve their emotional and physical well-being when dealing with anxiety and depression related to body image issues and self-esteem. Additionally, teens desired to have meaningful interactions with healthcare providers. Dowdy reported many accounts of teens feeling as though they were not being taken seriously or were not given adequate information regarding their health. Teens expressed frustration when a diagnosis of PCOS was delayed and when channels of communication between themselves and the healthcare provider were not seemingly open or accessible. As teens learned more about PCOS, Dowdy found concerns surrounding primary infertility began to rise. 
Other areas of concern were irregular menses which manifests in over 75 percent of those with PCOS. Often menstrual irregularities are treated with hormonal contraceptives, such as the birth control pill. Dowdy reported concern from teens regarding conversing with their mothers about the use of contraceptive pills to regulate menses. Concern and conflict arise when one or both parents are resistant to their daughter using birth control even when the reason is therapeutic. Parental concerns tend to involve uncertainty related to hormone exposure and adverse effects of the drug, negative perceptions of birth control pills from personal experience, fear the child will not adhere to the appropriate use of the drug, the possibility of the child believing sexual activity is permitted once on the birth control pill, and concern regarding others' perceptions of the child if they knew she was taking the birth control pill (Dowdy, 2012). An issue important to address when working with adolescents with PCOS is the impact on future fertility. Studies have shown adolescent girls are often very concerned about the threat of infertility and the impact of PCOS may have on their ability to conceive (Dramusic, Rajan, Chan, Ratman, \& Wong, 1997; Trent et al., 2002). If this aspect of PCOS is not adequately addressed with the adolescent, she may seek information elsewhere, feeling frustrated with a lack of communication with the provider, or the adolescent could become noncompliant with taking oral contraceptive pills as directed if she believes she cannot become pregnant with PCOS (Dowdy, 2012).

Dowdy (2012) also identified weight problems, acne, and hirsutism as areas of concern in adolescent girls with PCOS. These symptoms often lead to self-image problems which may negatively impact sexuality and hinder social interactions. In 
addition to addressing adolescents' concerns about their mothers' perceptions of using oral contractive pills to manage the symptoms of PCOS, Dowdy drew attention to the issue of weight and how often parents may become frustrated or fixated on their daughters' difficulties with weight management. This extenuates the issue of negative body image and the risk for disordered eating (Neumark-Sztainer, Story, Hannan, Perry, \& Irving, 2002). Dowdy urged healthcare providers, namely nurses, to engage this population of adolescent girls by listening and helping to provide the education and care needed in order to increase the quality of life of adolescent girls with PCOS.

Regarding quality of life specifically, Trent et al. (2002) conducted a hallmark study of adolescent girls with PCOS in effort to understand the relationship between PCOS and health-related quality of life. Holding the assumption adolescent girls are at a higher risk for quality of life disturbances given the nature of adolescence including heighted self-awareness, concerns regarding body image, desire for peer approval, and identity development, Trent et al. aimed to determine whether clinically or self-perceived severity of illness affects health-related quality of life in this population. Compared to healthy comparisons, the girls with PCOS demonstrated lower health-related quality of life scores in the areas of family activities, behavior, physical functioning, and general health perceptions (Trent et al., 2002). The girls perceived the severity of their illness as mild or moderate which was encouraging to the researchers as adolescents must cope with a chronic illness over an extended period of time. Importantly, it was noted the patient's perception of the illness had a greater impact on quality of life than the health care provider's perception of the illness; therefore, providers should be aware of the need 
to address social and developmental situations perceived as stressful to the adolescent in order to improve overall quality of life. Lastly, Trent et al. emphasized the importance of future research aimed at developing supportive interventions addressing quality of life in adolescents with PCOS.

Building on research surrounding quality of life in the adolescent population with PCOS, Harris-Glocker, Davidson, Kochman, Guzick, and Hoeger (2010) studied the effect of treatment with oral contraceptives and lifestyle changes with or without taking metformin on quality of life in obese patients. The study was a randomized placebocontrolled trial. Over a 24-week period, adolescents, along with a parent or guardian, attended educational sessions on healthy lifestyle changes and group exercise experiences. Participants were encouraged to decrease caloric intake by 500 calories daily and to exercise for 30 minutes daily. Data analysis showed as BMI was reduced, quality of life increased. Similarly, Trent, Austin, Rich, and Gordon (2005) assessed 97 adolescent girls with PCOS and found BMI to be inversely related to quality of life. In the study conducted by Harris-Glocker et al. (2010), the severity of hirsutism was also strongly correlated with decreased quality of life. Overall, obese adolescents with PCOS treated with oral contraception and lifestyle modification had improved quality of life regardless of treatment with metformin. Thus, Harris-Glocker et al. (2010) and Trent et al. (2005) recommended healthcare providers be attentive to specific adolescent concerns surrounding PCOS and offer plenty of support and guidance in achieving optimal health and wellness goals. 
Upon examining the health-related quality of life from a qualitative perspective, Jones et al. (2011) espoused the need for providers to more thoroughly evaluate the emotional well-being of adolescents with PCOS. Interviews with 15 girls, ranging in age from 17 to 21 years old, revealed weight problems and body perceptions as the most significant contributors to decreased health-related quality of life. Weight problems including unexplained weight gain, inability to lose weight, fluctuating weight, and distribution of weight were all concerns expressed by participants. The girls' body perceptions were shown to negatively impact self-confidence, emotional well-being, social functioning, and sexual relationships (Jones et al., 2011). Additionally, symptoms of hirsutism and acne led to poor self-image and fears and concerns related to future fertility also reduced emotional quality of life. Many of the concerns adolescents had were related in some way to the lack of information they had received regarding PCOS from their health care providers. Therefore, Jones et al. (2011) urged providers to devote time to foster the emotional well-being of adolescent girls with PCOS ensuring proper health education and open lines of communication.

Weiss and Bulmer (2011) also reported frustration from young women regarding care received from healthcare providers. Interviews with participants revealed a theme surrounding patient-provider relationships which involved participants expressing a need for more providers to be more knowledgeable about PCOS, to diagnose the syndrome sooner, and to more thoroughly explain why certain treatments or lifestyle modifications are necessary. Similar to other studies, the most immediate concerns expressed by the participants were related to appearance. Again, this finding is consistent with the 
understanding that adolescents are at a period of life in which body image and selfesteem issues are paramount. Weiss and Bulmer also identified a theme of concerns for future self. The young women in the study articulated concern over developing chronic health conditions and possible infertility. Interestingly, the researchers identified a stronger concern over infertility in the adolescent population when compared to older counterparts according to the literature. Hahn et al. (2005) proposed a reason for greater concern over infertility in the adolescent population as compared to older women may be because the older women may have the support of a partner or spouse which positively impacts quality of life; whereas, adolescents are left with "what if" questions regarding infertility. One participant in the Weiss and Bulmer (2011) study conveyed her concern over whether or not her potential infertility may negatively impact a future marital relationship. In conclusion, Weiss and Bulmer recommended future research in order to better understand and improve the psychosocial functioning of adolescents with PCOS.

Finally, one study evaluating the effectiveness of an intervention to improve physical and emotional disturbances in adolescent girls was found. Recognizing poor psychosocial functioning of adolescent girls with PCOS is associated with suboptimal medical outcomes, increased mortality, and higher healthcare costs Rofey et al. (2009) studied the effectiveness of a cognitive-behavioral therapy intervention in a group of 12 females with a mean age of 15.7 years. This pilot study showed statistically significant reductions in depression and obesity among the participants after implementing the Primary and Secondary Control Enhancement Training for Physical Illness (PASCETPI). The intervention included both individual and family sessions consisting of 
discussion of selected topics related to PCOS, weigh-ins and BMI calculation, monitored physical activity, and family communication. In addition to achieving weight loss and reducing depression, other physical comorbidities were positively impacted by the intervention including regularity of menses, blood pressure, sleeping disorders, waist circumference. Rofey et al. (2009) espoused adolescence as a critical time in developing one's regulatory processes and autonomous motivational systems. Therefore, the application of cognitive-based therapy interventions in adolescents with PCOS should be further explored as these girls are at a time in life where they are able to actively develop habits and skills in order to greatly improve their future health and quality of life (Rofey et al., 2009).

\section{Adolescent Coping}

While there were no studies found in the existing literature examining coping strategies or behaviors in adolescents with PCOS specifically, relevant articles exploring coping using Lazarus and Folkman's theory of stress and coping in children and adolescents were reviewed. Additionally, a study investigating the ways of coping in adolescents with schizophrenia was found to inform the current study in respect to the conceptual framework.

First, Compas (1987) conceptualized coping during childhood and adolescence based on the work conducted by Lazarus and Folkman (1984). Compas summarized the substantial extent to which the cognitive and behavioral coping efforts of children and adolescents affect their responses to stress. He espoused the ability children and adolescents have to cope using both problem-focused and emotion-focused coping 
strategies as delineated by Lazarus and Folkman (1984). Regarding the effectiveness of certain coping strategies in a given situation, Compas (1987) noted the impact of perceived personal control by the child or adolescent. For example, when faced with a situation perceived to be beyond control, coping cognitively by reframing the situation may be effective. On the other hand, in situations where the child or adolescent perceives a considerable amount of control, coping through emotional regulation may be more effective than actually doing something to relieve the stress which would be considered problem-focused. Regardless of how a child or an adolescent copes with stress, supportive parents or guardians were found to be important resources for facilitating coping. Additionally, high self-esteem was determined to positively affect one's ability to cope with stress. Finally, variables such as gender role identity and sexual socialization were noted as to potentially impact coping styles and strategies among boys and girls; therefore, Compas (1987) called for future research investigating the relation between various social contexts and coping behaviors of children and adolescents.

In 1988, Compas, Malcarne, and Fondacaro studied 130 boys and girls ranging in age from 10 to 14 in order to determine the capacity participants possessed to generate alternative coping solutions when faced with stressful situations and the actual coping strategies used to manage stress related to interpersonal or academic conflicts. Just as Compas (1987) reported the relationship between perceived control and coping strategies, Compas et al. (1988) found significant relationships between the two variables. In this study, the application of problem-focused strategies when dealing with a situation perceived as controllable to some degree was associated with less behavioral problems. 
Conversely, generating problem-focused alternatives when little to no control was perceived was related to higher behavioral problems. Notably, problem-focused strategies in general were found to be more developed in children and adolescents than emotion-focused strategies when compared to the adult population. Analysis of the data showed an increase in the use of emotion-focused coping strategies from the sixth to eighth grade, whereas, the use of problem-focused coping remained relatively unchanged. Also, girls were more likely to use emotion-focused coping strategies when faced with academic stressors than their male counterparts. Overall, Compas et al. (1988) indicated the usefulness of applying the stress and coping theory developed by Lazarus and Folkman (1984) when studying coping processes and specific coping strategies in children and adolescents.

Again applying the Lazarus and Folkman (1984) framework for stress and coping, Lee and Schepp (2011) investigated the ways in which adolescents with schizophrenia cope with stressful life events. A sample size of 40 male and female adolescents with schizophrenia between the age of 15 and 19 were enrolled in the study. A sample of 30 healthy controls were also selected for participation in the study. While schizophrenia and PCOS are very different illnesses and coping strategies identified in the group with schizophrenia cannot be generalized to girls with PCOS, this study does inform the current study by examining the relationship between problem and emotion-focused coping strategies and perceived control on overall health outcomes in adolescents. The findings from this study supported problem-focused coping as being positively associated with perceived personal control. Emotion-focused coping was determined to be effective 
for short-term coping in order to reduce the impact of stress; however, problem-oriented coping strategies were recommended as the primary method for long-term coping. Problem-oriented coping was related to more positive health outcomes overall. In summary, Lee and Schepp (2011) emphasized the importance of the healthcare provider' role in teaching adolescents with schizophrenia ways to manage stress and their response to stress in addition to helping them gain a sense of control over their mental illness. Given the evidence of the impact of perceived control on an adolescent's ability to cope, providers caring for girls with PCOS should also consider ways to enhance perceived control over the symptoms in order to positively influence health outcomes. Influence of Mother-Daughter Relationships on Body Image and Self-Esteem

The current study examined adolescent perceptions of maternal stress and controllability of components associated with PCOS on coping and depression. The existing literature was reviewed in effort to support this area of interest, however, there were no studies found specifically exploring the impact of mother-daughter relationships on coping with PCOS or studies observing the effect of PCOS on mother-daughter relationships. Nevertheless, two relevant studies were identified supporting the impact of mother-daughter relationships on variables including body image, self-esteem, and gender role identity (Usmiani \& Daniluk, 1997; Izydorczyk, 2010).

Usmiani and Daniluk (1997) noted the importance of body image in the development of one's identity during adolescence. Menarche marks the beginning of a new phase of life, challenging one with psychosocial and physical changes. During this major developmental transition, girls reorganize perceptions surrounding body image and 
sexual identity. Faced with cultural ideals of body types and increased self-awareness, adolescent girls are at a very high risk for low self-esteem and negative body image (Usimiani \& Daniluk, 1997). Studies have validated the impact one's family has on selfperceptions and impact on lifestyle habits. Mothers often serve as role models and sources of information for their adolescent daughters (Usimiani \& Daniluk, 1997; Rofey et al., 2009). Therefore, Usimiani and Daniluk surveyed 82 mothers and their menstruating daughters and 32 mothers of girls who had yet to begin menstruating. The girls included in the study were between the ages of 11 and 16 years. The researchers found higher self-esteem was associated with more positive body image in mothers and menstrual daughters. Additionally, there was a strong correlation between body image in mothers and daughters. When the mother's self-esteem rose, the daughter's self-esteem was also found to be higher. The observed correlations supported the importance influence mothers have on their daughter's development of body image and self-esteem during puberty. However, due to limitations including a relatively homogeneous sample and potential sampling bias, Usimiani and Daniluk recommended further exploration into the phenomenon of maternal influence on self-esteem and body image in their adolescent daughters.

Isdorczyk (2010) approached the issue of body image among adolescent girls suffering from anorexia nervosa. The study was aimed at comparing the body image of the girls with the body image of their mothers to determine any correlations. Participants were evaluated based on ratings of their perceptions of their actual self, ideal self, and ought self, that is what the participant believes she should look like. There was a positive 
correlation between the mothers' ideal self-beliefs and the desire to achieve the ideal shape and those of their daughters. The observed trend was for daughter to follow suit when her mother possessed a stronger desire to conform to cultural and social ideals for thinness. The correlations between mothers' and daughters' perceptions of self were obviously related, however, Isdorczyk questioned the direction of the influence. She offered that the adolescent's constant obsession with weight and weight loss could cause the mother to become more self-conscious of her current weight. Conversely, the mother's tendencies and self-perceptions could influence the daughter's identity development. Regardless of the direction of the relationship, mother-daughter relationships are influential with respect to body image. Given the importance of selfimage on one's ability to cope and avoid mood disorders such as depression, there is a need for further investigation into the perceptions of mothers and daughters in situations that threaten one's appearance such as PCOS.

\section{Inferences for Current Study}

A gap in the literature is evident considering the dearth of quantitative and lack of mixed methods studies examining coping and depression in adolescent girls with PCOS. Stressors associated with PCOS including irregular menses, hirsutism, acne, and weight problems have been well documented; however, the degree to which adolescent girls perceive control over these stressors and their perceptions of their mothers feelings regarding the symptoms of PCOS have not been evaluated in the existing literature. Furthermore, specific ways of coping in this population have not been examined. While existing literature supports an increased risk for mood disorders in adolescent girls with 
PCOS and maladaptive coping strategies have been linked to psychiatric morbidity, the relationship between ways of coping and depression scores in adolescent girls with PCOS has not been studied (Trent et al., 2002; Benson et al., 2010). Therefore, the need for the current study is supported by the literature, and the findings of this study will contribute substantially to the body of knowledge surrounding the psychosocial care of adolescents with PCOS.

\section{Summary}

This chapter provided a review of the current literature related to adolescents with PCOS including issues related to diagnosis and management, mother-daughter relationships, the prevalence of mood disorders, and coping. Additionally, supporting literature examining the psychosocial implications of PCOS in adult women was also discussed. Finally, inferences for the current study were presented based on the synthesis of literature. 


\section{CHAPTER THREE}

\section{METHODOLOGY}

This chapter provides a discussion of the research methodology for the study including an explanation of the convergent parallel mixed methods design and justification for using this design to address the purpose of the study. In addition to the

design, the setting and sample will be outlined. A review of the instrumentation and procedures will be addressed, followed by an overview of the plan for data analysis. Finally, issues of rigor, potential limitations, and protection of human subjects will be presented.

\section{Research Design}

The purpose of this study was to examine coping and depression in adolescent girls with PCOS. In order to address the phenomenon of interest, a convergent parallel mixed methods approach was utilized. By definition, the convergent design involves collecting and analyzing both quantitative and qualitative data during the same phase, then merging and interpreting the data which provides a more comprehensive understanding of the phenomenon of interest (Creswell \& Plano Clark, 2011). As in this study, the most common variant of the convergent design is the parallel-databases approach. Thus, the two forms of data obtained with the quantitative and qualitative strands were brought together only during the interpretation phase. The qualitative 
sample was smaller than the quantitative sample as the aim of the qualitative sample was to produce a rich, comprehensive understanding of the experiences of adolescent girls with PCOS, whereas quantitative data provides the basis to make generalizations to the population (Creswell \& Plano Clark, 2011).

The overall purpose of the convergent parallel design is better understanding of the research problem by obtaining both different but complementary data on the topic (Creswell \& Plano Clark, 2011). Collecting both forms of data during the same phase allowed the girls to reflect upon their answers on the questionnaires and engage in interviews soon thereafter. Both forms of data were important in understanding the ways in which adolescents cope with PCOS. Quantitatively evaluating the variables of perceived control, stress, maternal stress, coping, and depression along with hearing the voices of these girls to further explain their perceptions and situations contributing to their quantitative scores enhanced what is known about adolescent girls' experiences coping with PCOS.

This study began with a descriptive study in which data were collected using questionnaires. The girls completing the questionnaires were asked to indicate whether or not they were willing to participate in an interview regarding their experiences with PCOS. From those indicating willingness to participate, individual semi-structured interviews were conducted with a purposively selected subset of the girls. While addressing the quantitative research questions utilizing only the descriptive study was adequate to describe the frequency of scores for certain variables and relationships among them, the quantitative study alone was not adequate to explain the particular experiences 
of adolescent girls and how those experiences influence their perception of stressors, coping behaviors, and depression. Therefore, the interviews allowed for a deeper understanding of the individualized experiences of adolescent girls with PCOS and were used to validate relationships observed in the quantitative data (Creswell \& Plano Clark, 2011). In line with the intent and appropriate use of the convergent parallel design, the collection and analysis of data were concurrent but separate. The results from each strand were independent and are equally important in addressing the overall purpose of the study when merged and interpreted. Thus, together the findings from both strands best addressed the purpose of the study and enhanced the overall understanding of the phenomenon of interest (Creswell \& Plano Clark, 2011).

Finally, in congruence with the convergent mixed methods design, the philosophical assumptions of pragmatism guided the study (Creswell \& Plano Clark, 2011). A pragmatic worldview holds both subjective and objective knowledge in regard, rejecting postpositivists' and constructivists' viewpoints which require a phenomenon to be evaluated using either an inductive or deductive method. Considering the equal importance of the quantitative and qualitative data in addressing the research question, the pragmatist paradigm acts as a foundation supporting the practicality of addressing the research questions with multiple forms of data collection (Polit \& Beck, 2012). Fundamental to the pragmatic paradigm is the assumption that the research question or purpose of the study should drive the study, rather than the philosophical underpinnings. Thus, the most appropriate paradigm to guide the convergent mixed methods study was pragmatism (Creswell \& Plano Clark, 2011). 
To practically address the phenomenon of interest, it was important to recognize how greatly individual differences influence one's perception of the environment and how one copes with these stressors (Lazarus \& Folkman, 1984). Therefore, while the quantitative data provided information to discover, describe, and explain the phenomenon of interest, interacting with the participants to gain understanding of their experiences with PCOS substantially enhanced the quantitative findings by providing multiple individual perspectives. Comparing the results from each strand allowed for the building of new meaning and insight into the phenomenon of interest. Finally, in accordance with best practices in mixed methods research according to Creswell \& Plano Clark (2011), Figure 2 is provided as a clearly depicted procedural diagram in an effort to provide clarification regarding the complexity of the convergent parallel mixed methods design. 


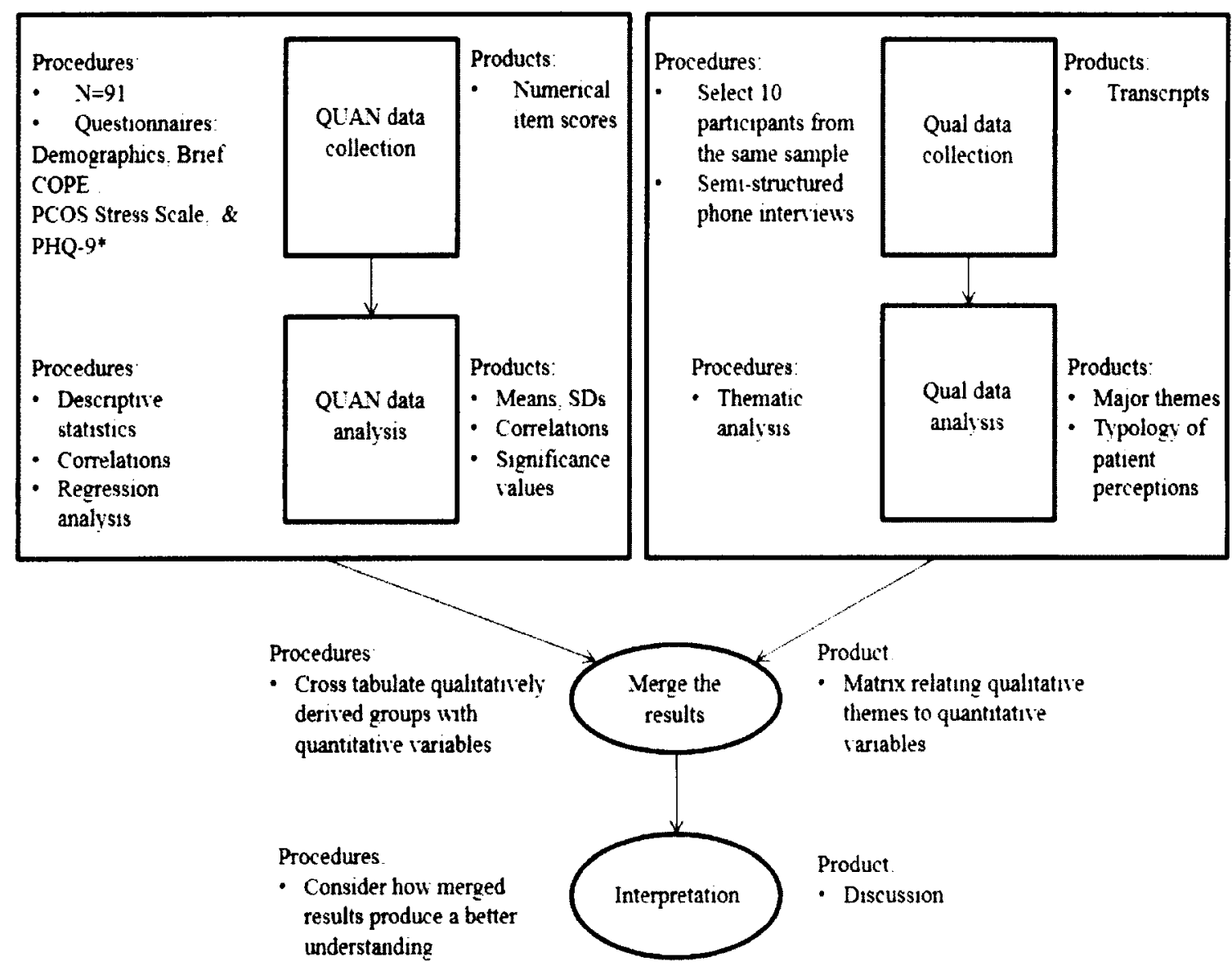

- Patient Health Questionnaire-9

Figure 2. Convergent parallel design diagram for study

\section{Setting and Sample}

The study was conducted within an urban academic healthcare system in the southeastern region of the United States. Participants were recruited from clinical practices within the healthcare system including practices specializing in pediatric and 
adolescent gynecology, pediatric endocrinology, and pediatric obesity management. Participants were selected for participation in quantitative phase of this study using a convenience sampling method. Although this nonprobability sampling method limited the generalizability of the study findings due to the limited representativeness of the sample, convenience sampling at one institution was the most practical way to potentially recruit a sufficient sample size (Polit \& Beck, 2012). However, to enhance the generalizability of the sample, the researcher did recruit participants from different medical practices within the healthcare system. In doing so, the findings represented participants who varied socioeconomically and who were receiving care from different healthcare providers. Therefore, the researcher was able to capture variance in the sample by recruiting participants from various locations (Polit \& Beck, 2012).

To be considered for inclusion in the study, girls had to be between the ages of 13 and 18 years, and must have had a diagnosis of PCOS as per the Rotterdam PCOS Consensus Group (2004). Alternatively they could have met the Rotterdam criteria as exemplified by documentation in the medical record of two of the three following conditions: 1) a laboratory diagnosis or confirmed clinical signs of hyperandrogenism, 2) ovarian morphology consistent with PCOS on ultrasound, and/or 3) menstrual irregularity. Additionally, girls had to be able to read and speak English. Exclusion criteria included a current pregnancy or a current medical situation including acute physical illness or psychological crisis. Any known cognitive delays or difficulties with language that would prevent the girl from being able to successfully complete the questionnaires or interview were considered reasons for exclusion from the study. 
Lastly, girls having been diagnosed with hyperprolactinemia, adrenal hyperplasia, or a diagnosed or suspected androgen-secreting tumor were excluded from the study.

The targeted sample size for the quantitative strand of this study was based on quantitative research question 5 , which was anticipated to involve 5 independent variables. In the power analysis, a sample size of 91 participants was targeted as it would have provided a power of .80 to detect an effect size for multiple $R\left(f^{2}\right)$ of .15 with an alpha of .05. For the qualitative strand of the study, a purposive sub-sample of the girls from the quantitative strand, reflecting demographic differences, was selected to best represent the entire group of girls (Creswell \& Plano Clark, 2011). The demographic differences in the sample accounted for age and race. Both younger and older adolescent girls were selected to participate in the interviews in order to reveal any possible differences in experiences with PCOS related to age. Additionally, girls of different races and ethnicities were interviewed to account for potential differences in experiences with PCOS based on racial or cultural differences. A sample size of approximately ten girls, less or more depending on data saturation, was projected for the qualitative sample (Streubert \& Carpenter, 2011).

\section{Instrumentation}

In addition to demographic information, four self-report instruments were used to collect data for the quantitative strand of the study. Qualitative data were collected using a semi-structured interview guide. This section will describe each instrument and the interview guide. Copies of the instruments are included in the Appendices. 
Demographic Characteristics

A demographic data form was included in the packet of questionnaires distributed to each participant. Participants were asked to complete the form, providing their age, age at diagnosis with PCOS or documented diagnoses which meet the Rotterdam PCOS criteria, ethnicity, race, and the insurance status as a proxy for socioeconomic status. A list of diagnoses meeting the Rotterdam PCOS criteria was provided on the form for the participants to reference so they can best answer the question regarding age of diagnosis (see Appendix A for the complete demographic form).

PCOS Stressor Scale

A researcher developed questionnaire was used to evaluate the stress and concern associated with aspects of PCOS, the degree of control the adolescent girls perceive to have over the stressors, and their perceptions of maternal concern related to the aspects of PCOS. The questionnaire consisted of three sections composed of items measuring each of the three factors: perceived personal concern, perceived control, and perceived maternal concern. The first section of the questionnaire asked the girls to rate perceptions of concern for each potentially stressful component of PCOS including hirsutism, weight concerns, irregular periods, the threat of chronic disease, threat of future infertility, and acne on a Likert scale from 0 "does not concern me at all" to 3 "concerns me very much". Possible range for the total score was 0 to 18 with higher scores indicating greater perceived concern. For the same items, the girls were asked to indicate the level of control they perceive over each component on a scale of 0 "nothing 1 can do to control it" to 3 "totally within my control". Possible range for the total score on 
perceived controllability was 0 to 18 with higher scores indicating higher perceived control. Finally, the girls were asked to indicate the degree to which they perceive their mother was concerned regarding each of the same items on a scale of 0 "my mother is not concerned at all" to 3 "my mother is very concerned." Again, the possible total score on perceived maternal concern ranged from 0 to 18 with higher scores indicating greater perceived maternal stress. It is important to note for purposes of interpreting findings that higher scores on the perceived controllability subscale were conceptually positive, indicating greater control, whereas, higher scores on the perceived personal concern and perceived maternal concern subscales were conceptually negative, indicating greater concern. A copy of this questionnaire is included in the Appendices (Appendix B). Coping Behaviors

Coping behaviors of the participants were measured with the Brief COPE developed by Carver (1997) (Appendix C). Adapted from the COPE inventory developed by Carver, Scheier, and Weintraub (1989), the Brief COPE is a 28-item questionnaire composed of 14 scales containing two items each. The stress and coping theory of Lazarus and Folkman (1984) served as the conceptual basis for the development of COPE inventory and the Brief COPE. Carver (1997) created the brief inventory to eliminate redundant items and to provide a more succinct option that would be easier and less time consuming for research participants to complete.

The 14 scales measure distinctively different coping strategies, some of which are adaptive and others that are maladaptive or problematic. Coping strategies such as the use of drugs and/or alcohol, denial, self-blame, planning, and positive reframing are 
examples of reactions addressed on the Brief COPE questionnaire. Participants were asked to respond to the statements based on how they would respond to a stressful event. In this study, the questionnaire was oriented to the stressors of having PCOS. There are a total of 28 , four-point Likert scale items. The possible range of scores for each subscale was 2 to 8 . Higher scores indicated greater use of the particular coping strategy, whereas, lower scores indicate less use of the strategy. Originally validated and shown to be reliable based on a study of 168 adults having experienced a natural disaster, the internal consistency reliability of each scale included in the Brief COPE was supported with alpha coefficients ranging from 0.50 to 0.90 (Carver, 1997). Construct validity was determined by an exploratory factor analysis which revealed nine factors with eigenvalues greater than 1.0. These factors accounted for 72.4 percent of the variance in responding. The Brief COPE has also been determined to be useful in assessing adolescent populations in several studies (Townsend, 2002; Wilson, Pritchard, \& Revalee, 2005; Brytek-Matera, 2007).

Depression

The Patient Health Questionnaire 9-item (PHQ-9) (Appendix D) tool was used to measure depressive symptoms. The PHQ-9 is a self-reported, easily interpreted, simplyscored, brief screening tool for depression. The PHQ-9 was adapted from the full Patient Health Questionnaire (PHQ) which consists of three pages and addresses eight different diagnoses (Kroenke, Spitzer, \& Williams, 2001). The tool has been validated for use in detecting major depression in the adolescent population (Richardson et al., 2010). The PHQ-9 is made up of nine, four-point Likert scale items. Scores range from 0 to 27 with 
higher scores indicating greater depression. To identify individuals with depression, typically the score must be higher than 10 . In a study of adults, $93 \%$ of participants with scores below 10 did not have depression, while $88 \%$ of participants scoring greater than 10 on the PHQ-9 were found to have major depression upon further evaluation (Kroenke et al., 2001).

In a study of 499 thirteen to seventeen year olds, of which $60 \%$ were female, the PHQ-9 was found to have an $89.5 \%$ sensitivity and a $78.8 \%$ specificity in detecting major depression in adolescents (Richardson et al., 2010). The internal consistency reliability of the PHQ-9 was supported with an alpha coefficient of 0.89 (Kroenke et al., 2001). Test-retest reliability was strong, demonstrating a correlation of 0.84 . Content validity was supported by a review of experts. Construct validity was demonstrated by correlating test items to measures for functional status, disability days, and symptomrelated difficulty. Finally, results from interviews with patients by mental health professionals demonstrated criterion-related validity of the PHQ-9 (Kroenke et al., 2011). Overall, psychometric analysis of the PHQ-9 supports the tool as a reliable and valid measure for depressive symptoms in adolescent girls.

Qualitative Data Collection

Qualitative data were generated utilizing a researcher-developed semi-structured interview guide which enhanced the depth of understanding surrounding participants' experiences with PCOS and allowed for further explanation of the quantitative results (Streubert \& Carpenter, 2011). The interview guide began with the grand tour question, "What is it like to have polycystic ovary disease?" 
Probes for the interview included: 1) "What is the most stressful thing about having PCOS?" 2) "Describe your sense of control over the symptoms you experience related to having PCOS." 3) "Describe your sense of control over potential negative health implications related to PCOS." 4) "What things do you do to help you cope with having PCOS?"5) "How has PCOS affected your relationship with your family and friends?" Probes used in the interview were informed by findings in the quantitative data. The complete interview guide is included in the Appendices (Appendix E).

\section{Procedures}

Prior to beginning data collection, approval from the Institutional Review Board (IRB) of Mercer University and the health care system from which participants were recruited was obtained. A copy of the Mercer University IRB approval is included in the Appendices (Appendix F). When possible, a face-to-face recruitment strategy was used as this method is typically more effective than recruiting through mail, phone calls, or electronic means (Polit \& Beck, 2012). A researcher developed flyer describing the purpose of the study and the inclusion and exclusion criteria was distributed to health care providers in each medical practice site included in the study. When patients who meet the criteria were being seen in the medical offices for visits, providers asked the adolescent girls and their parent or guardian if they were interested in participating in the study. Interested participants and their parents were asked to complete assent and consent documents and the study packet including the study questionnaires. The study packet included a place for the participant to indicate whether or not she was willing to be contacted for an interview. Participating clinical practices were supplied with study 
packets and were asked to provide the packets to any patient meeting the eligibility requirements. Participants were recruited face-to-face by health care providers. Each packet included a pre-addressed envelope with paid postage in order to ease the process of returning the surveys if the participant chose to take the packet home rather than completing the packet in the office. All participants completing the quantitative phase of the study received a five dollar gift card to a fast food restaurant in appreciation for their participation.

Immediately upon receiving completed study packets, participants having indicated willingness to participate in an interview were contacted via telephone for an interview. When the researcher was available in the medical practice and a participant indicated willingness to participate in an interview, the participant was asked if she would be willing to take part in a face-to-face interview rather than a telephone interview. Therefore, when circumstances allowed, the researcher interviewed participants face-toface. Additionally, if girls were interviewed in the office, their mothers or guardians were asked to leave the room, thus all of the participants interviewed during office visits were interviewed without their mothers being present. A researcher-developed semistructured interview guide (Appendix E), was used to guide the interviews. Each interview was completed within 20 minutes. The interviews were recorded and transcribed verbatim. The researcher also maintained field notes of participants' tone and disposition along with the researcher's feelings and perceptions. Pseudonyms were used on the transcripts rather than participants' names. 
Data Analysis Plan

This section summarizes the plan for the specific data analytic approaches and procedures used in addressing the quantitative research questions, qualitative, and mixed methods research questions. In accordance with the research design, Creswell and Plano Clark's (2011) steps for data analysis were employed. First, the quantitative and qualitative data were collected separately, but concurrently. Second, the data were analyzed independently using analytic methods appropriate for answering each research question. Third, the findings were compared and related using a side-by-side comparison for merged data analysis. Fourth, information from each database was specified and compared across the dimensions determined in step three. Fifth, comprehensive analysis of the quantitative and qualitative data comparisons were completed. Sixth, the comparisons were represented in the discussion, and finally, results were interpreted in order to determine how the research questions were answered using the quantitative, qualitative, and mixed data.

\section{Quantitative Questions}

All of the quantitative data obtained from the surveys were entered into an SPSS database for data cleaning and analysis. Both descriptive statistics and regression analyses were used to evaluate the patterns of relationships between the variables. A two-tailed alpha of $p<.05$ was used as the criterion for statistical significance. Research Question 1: What aspects of PCOS do adolescent girls with PCOS perceive as most stressful? To address this question, the planned analysis was to calculate the total score for items measuring perceived concerns about PCOS stressors from participant 
responses on the PCOS Stressor Scale (PCOSSS). However, because of issues with low reliability, which will be reported in chapter 4 , an individual score for each item on the perceived concern subscale were calculated. The participants' perceptions of concern associated with aspects of PCOS were characterized using descriptive statistics, including mean, median, mode, and standard deviation.

Research Question 2: What aspects of PCOS do adolescent girls with PCOS perceive as most controllable? Like the first research question, to address this question a total score was calculated from the participants' responses on the control subscale of the PCOSSS. Individual scores for each item representing different aspects of PCOS on the subscale were also calculated. The participants' perceptions of control concerning certain aspects of PCOS were characterized using mean, median, mode, and standard deviation. Research Question 3: What types and extent of coping behaviors do adolescent girls use to cope with PCOS? To address this question scores were calculated for each of the 14 scales on the Brief COPE questionnaire. Descriptive statistics including mean, median, mode, and standard deviation were used to describe the type and extent of coping behaviors used by the participants.

Research Question 4: Are adolescent girls' perceptions of their mothers' feelings regarding aspects of PCOS associated with the type and extent of coping behaviors used by adolescent girls with PCOS? The relationships among the participants' perceptions of maternal stress and the type and extent of coping behaviors used were analyzed using Pearson Product Moment correlations. 
Research Question 5: Are perceived stressors, perceived controllability, types and extent of coping behaviors, and adolescent girls' perceptions of their mothers' feelings regarding aspects of $P C O S$ predictors of depression in adolescent girls with PCOS? Multiple linear regression was used to address this question. To obtain a more parsimonious regression model, prior to conducting the regression analysis, an exploratory univariate correlational analysis was conducted to ascertain which of the Brief COPE subscales were related to depression. Only those subscales that were significant at a two tailed alpha of $\mathrm{p}<.10$ were included in the final regression model. The dependent variable, depression, was measured with the total score from the PHQ-9. Along with the statistical significance of the model $F$ and multiple $\mathrm{R}$ squared, the direction and significance of the beta weights of the individual predictors were explored to understand the relationship between the independent variables and the outcome variable.

\section{Qualitative Question:}

Research Question 6: What are the experiences of adolescent girls in coping with the stressors associated with PCOS? This research question was addressed with the qualitative data obtained from the participant interviews. First, a professional transcriptionist was hired to transcribe the interviews verbatim into a Microsoft Word document. The transcriptions were checked for accuracy. The coding process began with in vivo coding using the actual words spoken by the participants (Saldaña, 2013). As adolescents' voices are often marginalized, using the actual words of the adolescent girls will provide for a deeper understanding of their perspectives (Saldaña, 2013). 
Descriptive and emotion codes were used to analyze the data to help make explicit the implicit meaning of the participants' words. Descriptive coding was useful in identifying topics discussed during the interviews, while emotion coding added depth to the exploration of participants' lived experiences by labeling expressed or inferred feelings (Saldaña, 2013). Having coded the transcripts with three type of first cycle codes, the data were clustered and observed for the emerging themes. Second cycle coding involved grouping the codes into broader themes and subthemes in preparation for comparing and interpreting the results when the quantitative and qualitative data are merged (Saldaña, 2013).

\section{Mixed Methods Question}

Research Question 7: To what extent do the quantitative results about stressors, perceived control, coping, and depression converge with the interview data reporting the coping experiences of adolescent girls with PCOS? Due to the primary purpose of the convergent parallel study being to obtain both quantitative and qualitative data in order to best understand the experiences of adolescent girls with PCOS, the data were compared and merged during the data analysis phase. The steps discussed above as outlined by Creswell and Plano Clark (2011) were followed in analyzing the data to answer the mixed methods question. The final step of interpretation involved a discussion of the ways in which the two types of data converged, related to each other, and produced an overall greater understanding of the phenomenon of interest (Creswell \& Plano Clark, 2011). 
Additionally, to ensure the reliability of the study findings, Lincoln and Guba's (1985) guidelines for achieving trustworthiness were followed. Credibility, dependability, confirmability, and transferability were safeguarded in order to implement and conduct a rigorous qualitative study. First, the convergent parallel design provided for triangulation of data in order to establish credibility. The quantitative and qualitative data complemented each other and were used for comparing and contrasting, thus enhancing the validity of findings (Creswell \& Plano Clark, 2011). Second, dependability was established through the process and product of the research (Lincoln \& Guba, 1985). The clear focus and plan in conducting the study according to the Creswell and Plano Clark's (2011) guidelines for convergent parallel studies and producing valid results and interpretations that are reflected in the data, having been confirmed by quality checks, ensured dependability. Confirmability, or objectivity, of the study was obtained by keeping an audit trail (Lincoln \& Guba, 1985). Detailed records were kept of the study methods and procedures used in conducting the research to provide a complete picture of how data were collected, processed, analyzed, and merged. Finally, transferability involves the extent to which the results of the study are generalizable. To help assure readers will be able to determine the usefulness and transferability of the results, a thick description of the data was provided.

\section{Protection of Human Subjects}

Measures were taken to ensure the protection of the individuals participating in this mixed methods study. First, the study was approved by the IRB of both the researcher's academic institution as well as the institution where the study took place. 
The study packet included full disclosure to inform the girls and their parents or guardians of the study purposes and the methods for collecting data in order to inform their decision to participate. The provider distributing the study packets were sure to convey that this was a voluntary study. Assurance of confidentiality of information and the voluntary nature of the study were indicated on the packet the girls received. Care was taken in presenting the information regarding the study to the adolescent girls and their parents or guardians, treating them as autonomous agents, to ensure they understood their participation was voluntary and that they were able to withdraw at any time (Beauchamp \& Childress, 2013). For study participants under the age of 18, informed consent was obtained from their parent or legal guardian. Considering the 13 to 17 year old girls were mature enough to understand the basic informed consent information, their written assent was also obtained. Requesting assent from the participants demonstrated respect for their autonomy and self-determination (Polit \& Beck, 2012).

Careful attention was given to the protection of participant privacy. Participants were identified using pseudonyms and personal identifiable information was not recorded on any of the questionnaires, interview transcripts, demographic information forms, or any other documentation. All completed questionnaires, demographic forms, and interview transcripts were stored in a locked drawer in the researcher's office. The research information was not accessible to anyone other than the researcher and those directly involved in the research process. Finally, all electronic data was stored on a fire walled, password protected computer. 


\section{Summary}

This chapter has described the methodology for the convergent parallel mixed methods study including the design, sample and setting, and procedures. Detailed explanations of the instruments to be used for data collection were presented as well as an overview of how data will be analyzed. Finally, a description of the process for protecting human subjects was provided. 


\section{CHAPTER FOUR}

\section{RESULTS}

This chapter presents the data from this convergent mixed methods study examining coping and depression among adolescent girls with PCOS. Characteristics of the sample, data cleaning methods, and psychometrics of the quantitative instruments are delineated. Finally the quantitative, qualitative, and mixed methods results are presented for each research question.

\section{Overview of Data Analysis}

Quantitative data analysis was conducted using SPSS version 24.0. The data were entered into SPSS by the researcher and were screened for errors and cleaned using established protocols (Pallant, 2013). Frequencies were run on each variable to check minimum and maximum scores and to assess valid and missing data. If more than $20 \%$ of the items were missing for any instrument, no score was imputed and the participant's score was noted as missing. Missing data were minimal; however, in instances when data were missing, mean substitution was used to replace the missing value by calculating the mean score based on valid responses on the given subscale (Bannon, 2013). Tests of normality were conducted for the continuous variables and all variables were checked for any violation of the assumptions underlying the planned statistical analyses (Pallant, 2013). No violation of statistical assumptions were noted. 
Qualitative data of participants who completed interviews were transcribed verbatim by a professional transcriptionist into a Word document and checked for accuracy. The process for qualitative data analysis will be described in detail in the section discussing research question six.

\section{Description of the Sample}

Data were collected from January 1, 2016 until May 31, 2016. A sample of 91 girls was targeted. During the course of the study multiple challenges with recruitment resulted in the inability to recruit the proposed sample size, these included difficulty identifying adolescents with PCOS. Interestingly, during the period of recruitment the North American Society for Pediatric and Adolescent Gynecology (NASPAG) held their annual conference. PCOS was the main topic for two presentations at the meeting, and both discussions addressed the controversies and difficulties with diagnosing PCOS in adolescents (Bonny \& Javed, 2016; Bonny \& Gomez-Lobo, 2016). As addressed in chapter 2, the diagnosis of PCOS in adolescents is difficult as physiologic changes of puberty may overlap with clinical manifestations of PCOS (Agapova et al., 2014). Bonny and Gomez-Lobo (2016) discussed the risks of over-diagnosis in the adolescent including unnecessary labeling, unwarranted interventions, and overall impact on the adolescent's quality of life and anxiety. While there remains no consensus guidelines for the diagnosis of PCOS in the adolescent, the recommendation was made to take great caution before diagnosing adolescents with PCOS and engage in frequent longitudinal reevaluation (Bonny \& Gomez-Lobo, 2016). 
The diagnostic challenge with PCOS in this population was certainly observed during the recruitment phase of this study. The majority of healthcare providers were either hesitant or were not willing to distribute packets to girls without a documented diagnosis of PCOS even if they met the inclusion criteria for the study. While this factor severely limited the number of potential participants for this study, the healthcare providers' hesitations and concerns were valid and supported by the research presented at the NASPAG conference as they were protecting their patients from the risks associated with over-diagnosis, namely labeling and provoking unnecessary worry and anxiety in both the adolescent and the parents. Most healthcare providers at the clinical sites where this study was conducted would not distribute a research packet to the eligible girl until they had performed a physical examination, completed laboratory testing, made a diagnosis of PCOS, and counseled the girl and her parent(s) on the diagnosis. Often the eligible girl would not receive a study packet until her second or third visit to the office. Considering this delay, along with other factors, the pool of potentially eligible participants was restricted. Over a period of five months, approximately 35 girls who qualified for the study according to the inclusion and exclusion criteria were given a study packet during an office visit. Of those, a total of 23 girls (65.6\%) completed and returned the study packet. This difficulty with recruitment for this study demonstrates the evolving understanding of PCOS and the diagnostic process that was not fully realized when the study was proposed. 
Of the 23 participants who returned a completed packet, 18 indicated willingness to participate in an interview. Five of those 18 were interviewed face-to-face. The remaining 13 were contacted via telephone. If they did not answer, a voicemail message was left asking the participant to call the researcher at a convenient time. Six girls either answered when they were called for an interview or responded to the voicemail message by calling the researcher to complete the interview at a later time. Therefore, a total of 11 girls participated qualitative strand of the study.

All of the participants provided informed written consent and assent if the participant was a minor. The demographic characteristics of the sample are reported in Table 1. All of the participants had been diagnosed with PCOS, the majority of which were white adolescent girls. Most of the participants reported that they had experienced menstrual irregularities, high testosterone, and hirsutism. 
Table 1 Demographic Characteristics of Sample $(N=23)$

Variables

Age (Range 13-18)

Diagnosis of PCOS

$\begin{array}{lcl}\text { Yes } & 23 & (100 \%) \\ \text { No } & 0 & (0 \%)\end{array}$

Age of diagnosis with PCOS

Race

$\begin{array}{lcl}\text { White, non-Hispanic } & 14 & (60.9 \%) \\ \text { Hispanic } & 1 & (4.3 \%) \\ \text { Black } & 4 & (17.4 \%) \\ \text { Asian/Pacific Islander } & 0 & (0 \%) \\ \text { Multiracial } & 4 & (17.4 \%) \\ \text { Other } & 0 & (0 \%)\end{array}$

Ethnicity

Non-Hispanic/non-Latina

Hispanic/Latina

$22 \quad(95.7 \%)$

$1 \quad(4.3 \%)$

Health Insurance

Medicaid

Private insurance

Uninsured

Unknown

$\begin{array}{ll}9 & (39.1 \%) \\ 8 & (34.8 \%) \\ 0 & (0 \%) \\ 6 & (26.1 \%)\end{array}$

Experienced PCOS symptoms

Irregular periods

$23 \quad(100 \%)$

Hirsutism

$16 \quad(69.6 \%)$

High testosterone
Mean (SD)

\section{$16.2(1.4)$}
$15.1(1.5)$ 
Psychometric Properties of Study Instruments

Prior to conducting data analyses for each research question, the psychometric properties of the study instruments were examined. The researcher-developed PCOS Stressor Scale (PCOSSS) was used for the first time in this study. The PCOSSS consisted of three, 6-item subscales measuring girls' concerns, girls' controllability, and girls' perception of maternal concern. Cronbach's alpha was used to determine internal consistency reliability of all the instruments. Descriptive statistics for the instruments were used to address several of the research questions, thus the descriptive statistics are reported for each instrument when addressing individual research questions. Table 2 reports the internal consistency reliability for each study instrument.

Table 2 Internal Consistency Reliability of Study Instruments $(N=23)$

Instrument Cronbach's Alpha

Brief COPE $.81 \ddagger$

PHQ-9 .84

PCOSSS: Girls' Concerns $.11 \ddagger$

PCOSSS: Girls' Control $.71 \ddagger$

PCOSS: Maternal Concern $.62 \ddagger$

Note: $\ddagger n=22, \ddagger \ddagger n=21$; Patient Health Questionnaire 9-item (PHQ-9), Polycystic Ovary Syndrome Stressor Scale (PCOSSS). 
Using Nunnally and Bernstein's (1994) recommendation that the Cronbach's alpha should be .70 or higher, the Cronbach's alphas for the Brief COPE, the PHQ-9, and the PCOSSS Girls' Control scale were satisfactory. The PCOSSS Maternal Concern scale alpha was marginal, and the PCOSSS Girls' Concerns scale was not acceptable. Consequently, the items on the PCOSSS Girls' Concerns scale were included individually in analyses, rather than summing the items within a subscale. .

\section{Depression Among Adolescent Participants}

Participants' depression scores were analyzed using frequencies and descriptive statistics. During the five months of data collection, completed study packets were periodically reviewed to evaluate reported severity of depression. The majority $(56.5 \%)$ of the sample was at least moderately depressed with a score on the PHQ-9 between 10 and 24 . Of those, 46 percent were moderately severe or severely depressed. Five participants answered that they had thought about being better off dead or had thoughts of hurting oneself on "several days" over the past two weeks. One participant indicated that she had suicidal ideations "nearly every day" during the two weeks prior to completing the questionnaire. The researcher managed the depression scores by notifying the supervising physician at the research setting of any participant with at least moderate depression (a score of 10 or above on the PHQ-9) and those with indication of suicide. The supervising physician then contacted the participant's healthcare provider. The healthcare providers were informed of their patient's depression score or suicidal ideation 
and were asked to contact the patient's parent or guardian and to plan appropriate follow up care with the patient.

Data Analysis Addressing Research Questions

To address research questions one, two, and three, descriptive statistics were used. Research question four was addressed with Pearson product-moment correlations. A multiple linear regression analysis was used to address research question 5. Research question six was addressed through coding and identifying themes. Finally, the mixed methods research question seven was addressed by converging the quantitative data with the qualitative data.

Research Question 1- What aspects of PCOS do adolescent girls with PCOS perceive as most stressful?

Descriptive statistics for each item of the girls' concerns subscale of the PCOS Stressor Scale (PCOSSS) were used to address this question. These data are reported in Table 3. The possible range of scores on each item of this subscale was 0 to 3 with higher scores indicating higher levels of concern related to stressors associated with PCOS including acne, hirsutism, weight concerns, irregular periods, threat of chronic disease, and threat of future infertility. Participants reported weight concerns, irregular periods, and the threat of future infertility as the most stressful aspects of PCOS. The median score for all three of these items was 3.0 , reflecting the response "concerns me a lot" for the item. The low Cronbach's alpha for the scale suggested the concerns were not homogenous. Consequently a total score was not calculated for this instrument. 
Table 3 Descriptive Statistics for Girls' Concerns about PCOS Stressors ( $N=23)$

\begin{tabular}{lll} 
Variable & Mean (SD) & Median \\
\hline Acne & $1.55(0.96) \ddagger$ & 1.5 \\
Hirsutism & $1.83(1.19)$ & 2.0 \\
Weight concerns & $2.48(0.79)$ & 3.0 \\
Irregular periods & $2.30(0.88)$ & 3.0 \\
Threat of chronic disease & $1.59(1.18)^{\ddagger}$ & 2.0 \\
Threat of future infertility & $2.00(1.24)$ & 3.0
\end{tabular}

Note: $\ddagger n=22$.

Research Question 2- What aspects of PCOS do adolescent girls with PCOS perceive as most controllable?

Just as research question one was addressed, descriptive statistics were used to determine which aspects of PCOS participants perceived as most controllable. Table 4 summarizes the descriptive statistics for the girls' control subscale. Mirroring the subscale described for research question one, the girl's control subscale of the PCOSSS included items addressing six PCOS stressors: acne, hirsutism, weight concerns, irregular periods, threat of chronic disease, and threat of future infertility. The possible range of scores for each item on the subscale was 0 to 3 with lower scores indicating less control over the stressor. Participants reported perceiving the least amount of control over irregular periods, threat of chronic disease, and threat of future infertility with a median 
score of 1.0 for each item. A median of 1 for these items was notable as it indicated that $50 \%$ of the sample perceived they had "no control" to "little control" over the stressors. Additionally, the total score for the girl's control subscale was calculated. The possible range for the total score was 0 to 18 . The total score mean was 8.72 which indicated the participants perceived moderate control over stressors associated with PCOS.

Table 4 Descriptive Statistics for Girls' Control over PCOS Stressors $(N=23)$

\begin{tabular}{lll} 
Variable & Mean (SD) & Median \\
\hline Acne & $1.83(0.89)$ & 2.0 \\
Hirsutism & $1.36(1.22) \ddagger$ & 1.5 \\
Weight concerns & $1.70(0.97)$ & 2.0 \\
Irregular periods & $1.39(1.31)$ & 1.0 \\
Threat of chronic disease & $1.17(1.15)$ & 1.0 \\
Threat of future infertility & $1.13(1.06)$ & 1.0 \\
\hline Total score & $8.56(4.24)$ & 7.0 \\
\hline
\end{tabular}

Note: $\ddagger \mathrm{n}=22$. 
Research Question 3- What types and extent of coping behaviors do adolescent girls use to cope with PCOS?

To address research question three, scores on the Brief COPE instrument were analyzed using descriptive statistics. The Brief COPE consists of 28 individual items making up 14 subscales which represent different coping behaviors. According to the instructions for scoring the Brief COPE, two of the items were added to determine the total score for each of the 14 subscales (University of Miami, 2007). The possible range of scores for each subscale was 2 to 8 with higher scores indicating a greater use of the coping behavior. Participants could answer each item on the Brief COPE with a score from 1 to 4, with 1 indicating "I haven't been doing this at all" and 4 indicating "I've been doing this a lot." Thus, a score of 1 on an individual item on the questionnaire indicated the answer, "I haven't been doing this at all." Therefore, if a participant scored a 2 on any subscale, she had indicated she was not using that coping behavior at all. The types of coping behaviors and the extent to which they were used by participants are presented in Table 5. 
Table 5 Types and Extent of Coping Behaviors used by Girls with PCOS $(N=23)$

\begin{tabular}{lcll} 
Coping Behavior & $\begin{array}{c}\text { Observed Range } \\
\text { (Possible range 2-8) }\end{array}$ & Mean (SD) & Median \\
\hline Acceptance & $4-8$ & $6.83(1.27)$ & 7.0 \\
Self-distraction & $2-8$ & $5.87(1.36)$ & 6.0 \\
Positive reframing & $2-8$ & $5.43(1.90)$ & 5.0 \\
Active coping & $3-8$ & $5.04(1.52)$ & 5.0 \\
Planning & $2-8$ & $4.91(1.35) \ddagger$ & 5.0 \\
Use of emotional support & $2-8$ & $4.74(1.91)$ & 4.0 \\
Use of instrumental support & $2-7$ & $4.30(1.91)$ & 4.0 \\
Religion & $2-8$ & $4.26(2.22)$ & 4.0 \\
Self-blame & $2-7$ & $4.00(1.65)$ & 4.0 \\
Humor & $2-7$ & $3.78(1.57)$ & 4.0 \\
Venting & $2-6$ & $3.65(1.43)$ & 4.0 \\
Behavior disengagement & $2-8$ & $3.09(1.70)$ & 2.0 \\
Denial & $2-5$ & $2.61(1.03)$ & 2.0 \\
Substance use & $2-5$ & $2.35(0.83)$ & 2.0
\end{tabular}

Note: $\ddagger \mathrm{n}=22$.

Participants indicated the coping behavior of acceptance as being used to the greatest extent. Items for acceptance asked participants to indicate the extent to which 
they were accepting the reality of being diagnosed with PCOS and the extent to which they were learning to live with PCOS. With a median score of 7.0 for acceptance participants most frequently answered that they had "been doing this a medium amount" and/or had "been doing this a lot." Self-distraction with school, work, or other activities such as going to the movies, watching TV, reading, daydreaming, sleeping, or shopping was the coping behavior with the next highest extent of use. Substance use of was reported as the least used coping behavior.

Research question 4- Are adolescent girls' perceptions of their mothers' feelings regarding aspects of PCOS associated with the type and extent of coping behaviors used by adolescent girls with PCOS?

First, descriptive statistics for the perceived maternal concern subscale on the PCOSSS were analyzed and are reported in Table 6. The sample size was 22 for this analysis because one participant did not complete any of the items on the subscale measuring perceptions of maternal concern. The possible range of scores on each item of this subscale was 0 to 3 with higher scores indicating higher levels of perceived maternal concern related to stressors associated with PCOS including acne, hirsutism, weight concerns, irregular periods, threat of chronic disease, and threat of future infertility. A total possible score for the perceived maternal concern subscale was 0 to 18 with 0 indicating participants perceived their mothers were not concerned. Participants perceived their mothers to be the least concerned about acne and hirsutism. The median score for both acne and hirsutism was 1.0 indicating a response of "my mother is not 
concerned a little." The girls perceived a moderate amount of maternal concern related to weight concerns, irregular periods, threat of chronic disease, and threat of future infertility. The median score for both irregular periods and threat of chronic disease was 3.0 indicating the response, "my mother is concerned a lot." The total score for the subscale was calculated in addition to the score for each item. The total score mean was 11.64 indicating, as a whole, the participants perceived their mothers to be moderately concerned with the stressors indicated on the instrument.

When comparing girls' concerns (Table 3) with girls' perceptions of maternal concern (Table 6), there was congruence as the top three concerns the participants had and believed their mothers had were weight, irregular periods, and future infertility. Girls perceived their mothers as more concerned about irregular periods and future infertility than their own concerns. However, girls expressed more concern about their weight compared to perceptions of their mothers' concern. 
Table 6 Descriptive Statistics for Girls' Perceptions of Maternal Concern over PCOS

Stressors $(N=22)$

\begin{tabular}{lll} 
Variable & Mean (SD) & Median \\
\hline Acne & $1.09(0.92)$ & 1.0 \\
Hirsutism & $1.27(1.12)$ & 1.0 \\
Weight concerns & $2.23(0.87)$ & 2.0 \\
Irregular periods & $2.45(0.80)$ & 3.0 \\
Threat of chronic disease & $2.23(1.07)$ & 3.0 \\
Threat of future infertility & $2.36(0.79)$ & 2.5 \\
\hline Total score & & 11.5
\end{tabular}

To examine the relationship among perceived maternal concern and the type and extent of coping behavior used by participants, Pearson product moment correlations were used. The total score for the perceived maternal concern subscale on the PCOSSS instrument was correlated with each subscale score on the Brief COPE instrument. The results of the data analyses are reported in Table 7. 
Table 7 Pearson Product Moment Correlations between Girls' Perceptions of Maternal Concern and Coping Behaviors $(N=22)$

\begin{tabular}{lll} 
Coping Behavior & $\mathrm{r}$ & $\mathrm{p}$ value \\
\hline Self-distraction & .11 & .62 \\
Active coping & .21 & .33 \\
Denial & -.07 & .76 \\
Substance use & .15 & .50 \\
Use of emotional support & -.30 & .18 \\
Use of instrumental support & -.14 & .55 \\
Behavioral disengagement & .24 & .27 \\
Venting & -.12 & .58 \\
Positive reframing & -.06 & .80 \\
Planning & .20 & .37 \\
Humor & -.38 & .08 \\
Acceptance & $.50^{*}$ & .02 \\
Religion & -.28 & .20 \\
Self-blame & .10 & .65 \\
\hline
\end{tabular}

Note: ${ }^{*} \mathrm{p}<.05$ 
With a two-tailed alpha of 0.05 , one statistically significant correlation was observed. There was a moderate significant correlation between girls' perceptions of maternal concern and the use of acceptance as a coping behavior. Therefore, greater perceived maternal concern related to PCOS was associated with greater use of acceptance to cope with having PCOS. While the correlations were not statistically significant, there were weak inverse correlations observed between perceived maternal concern and emotional support, humor, and religion.

When examining the correlations between each item on the perceived maternal concern subscale and the coping behaviors, two statistically significant correlations were observed. Greater perceived maternal concern over irregular periods was significantly associated with greater use of self-distraction $(r=.43, p<.05)$ by participants. A significant negative association between maternal concern over hirsutism and use of humor $(r=-.50, p<.05)$ was observed; therefore, as participants perceived greater maternal concern over hirsutism their use of humor as a coping behavior decreased. Research question 5-Are perceived stressors, perceived controllability, types and extent of coping behaviors, and adolescent girls ' perceptions of their mothers' feelings regarding aspects of PCOS predictors of depression in adolescent girls with PCOS? Multiple linear regression was used to address research question five. Before running the regression analysis, an exploratory correlational analysis was conducted to ascertain which of the Brief COPE subscales were significantly related to depression. Only those subscales with a correlation significant at an alpha of $p<.10$ were included in 
the regression analysis. Because behavioral disengagement and substance use were not normally distributed variables a Spearman's rho correlation analysis was ran in addition to the Pearson product moment correlation to determine univariate significance. The same Brief COPE variables were significantly related to depression at $p<.10$ on both the Pearson product moment correlation and the Spearman's rho correlation analysis. Those variables were substance use $(r=.39)$, behavioral disengagement $(r=.64)$, religion $(r=-.42)$, and planning $(r=.36)$.

Additionally, due to the low Cronbach's alpha for the PCOSSS Girls' Concerns additional statistical analyses were conducted. First, the items on the girls' concerns subscale were dichotomized and recoded so that a score of 0 to 1 was recoded to a 0 and a scores of 2 to 3 was recoded to a 1 . The Kuder-Richardson alpha for the dichotomized items was still low, thus confirming the concerns items should not be summed for a total score. The new dichotomized items were then correlated with depression. Item number four on the subscale, which represented girls' concerns over irregular periods, was correlated with depression with an $\mathrm{r}$ of -.38 and a $\mathrm{p}$ value of .077 . Therefore, the dichotomized concern over irregular periods variable was included as an independent variable in the regression. The final regression model included the independent variables of girls' concern over irregular periods, girls' perceptions of control, perceptions of maternal concern, and the 4 coping subscales of substance use, behavioral disengagement, religion, and planning. The dependent variable was depression. Table 8 summarizes the results of the multiple linear regression analysis. 
Table 8 Results of Multiple Linear Regression Analysis of Perceived Stressors, Perceived Controllability, Types and Extent of Coping Behaviors, and Perceived Maternal Concerns as Predictors of Depression in Adolescent Girls with PCOS (N=23)

\begin{tabular}{lcc} 
Variable & Standardized beta weights & p value \\
\hline Girls' concern over irregular periods & -.286 & .224 \\
Girls' control & -.410 & $.049^{*}$ \\
Maternal concern & .050 & .792 \\
Substance use & .105 & .667 \\
Behavioral disengagement & .039 & .904 \\
Religion & -.452 & .103 \\
Planning & .173 & .510 \\
\hline R square & Adjusted R square & F \\
\hline .606 & .409 & $3.078^{*}$ \\
\hline
\end{tabular}

Note: ${ }^{*} \mathrm{p}<.05$

A statistically significant amount of variance (60.6\%) in depression was explained by the predictors $(F=3.078, p=.035)$. Girls' perceived control was a significant predictor of depression. Conceptually, as girls perceived less control over PCOS related stressors, their depression scores were greater. Maternal concern and coping behaviors were not significant predictors of depression. 
Research question 6- What are the experiences of adolescent girls in coping with the stressors associated with PCOS?

To address research question six, qualitative data were coded and analyzed for themes (Saldaña, 2013). First, the 11 interviews with the participants were transcribed verbatim into a Word document by a professional transcriptionist. Participants' names were redacted to maintain the confidentiality of the data. The researcher reviewed the transcripts for accuracy. First cycle coding began with three selected coding methods including, descriptive, in vivo, and emotion coding. Descriptive and in vivo coding are both elemental coding methods and serve to establish a foundation for future coding cycles. Descriptive coding provided a list of topics discussed during the interviews while the participant's own words were recorded through in vivo coding. In vivo coding was of particular importance as this method of coding is useful in hearing and understanding voices from marginalized populations, in this case, adolescent girls with PCOS (Saldaña, 2013). Emotion coding was also used in order to label the emotions expressed by the participant or inferred by the researcher (Saldaña, 2013).

After first cycle coding was completed, the researcher sent the initial coding to the dissertation chair for review. The dissertation chair also completed first cycle coding on the transcripts. To enhance the trustworthiness of the findings, the researcher and the dissertation chair discussed the evolving identified codes and determined there was consistency between the products from both first cycle coding processes. 
Through further analysis of the transcripts, the most salient themes and subthemes were identified. The coding process produced four themes and eight subthemes. The themes and subthemes will be discussed and illustrated with verbatim quotes. Listed below are the themes and supporting subthemes which derived from coding the transcripts from interviews with the participants.

Themes and Subthemes

1. Not knowing

a. What, why, how?

b. Others don't understand

2. Not in control

a. Weight concerns

b. Taking the medicines

3. Not now, not ever?

a. Irregular periods

b. Fertility concerns

4. Not horrible, not the best

a. Body image

b. Managing symptoms

Not Knowing

Throughout the interviews the phrases such as "I don't know," and "they don't understand" were repeated frequently by participants. While concerns about not knowing 
what the future holds in the short term with regard to irregular periods and in the long term concerning fertility and not knowing what to do about symptoms of PCOS are addressed in other themes because of their prevalence, this overarching theme of not knowing voiced by participants is important to highlight. Participants felt like they did not know what was going on with their bodies or how to manage their feelings, relationships, or symptoms. Moreover, they felt like others did not know what was going on with them. Consequently, a general feeling of not knowing was evident from the interviews. Therefore, two subthemes emerged from this theme: what, why, how? and others don't understand. Illustrating the theme of not knowing and the subtheme what, why, how, participants made the following statements when discussing their feelings about being diagnosed with PCOS: "When I first found out, like, I really didn't kind of believe it. It was just, like really embarrassing to be different from everybody else."; "I [didn't] know how it [had] affected me because...I never knew....the symptoms it could cause."; "If [I'm] struggling, [I] don't understand how everybody else has it so easy." Curious about her family history in regards to why and how she had come to have PCOS, one participant made the following statement:

...but I don't really know much about my father's side of the family and it makes me wonder, like have they had to deal with this. You know, because my dad's family most of the women are overweight, and you know, because l'm closer [to] what [they] look like.

Another girl expressed her feeling of not knowing with regard to her weight. When discussing her experience with PCOS symptoms she stated, "I don't know how I gained so much weight; I don't really eat much." This subtheme of what, why, how seemed to 
emerge from a general lack of knowledge about PCOS, genuine concern for oneself, and sometimes ambivalence about having PCOS. For example, when asked about her experience with PCOS said, "I haven't really felt any different. I think it's just not, like a normal period. They, um, did a blood sample, and then they, like, just, I don't know... [told me I had PCOS]."

In addition to a personal feeling of not knowing, the participants expressed feelings surrounding the "not knowing" of others around them. One participant expressed this challenge saying, "It's difficult being different from other people who just don't understand it." Another elaborated on this feeling of being different stating, "You feel like...you don't really fit in. Like you feel like you're the oddball out. You don't feel normal. You don't feel like everyone else around you." While several of the participants said they felt comfortable discussing things about PCOS with their mothers, many made statements about not talking about PCOS much in general, especially with their boyfriends, fathers, and friends. The underlying reason for not discussing PCOS with these individuals was because participants felt the men in their lives and their friends did not understand PCOS and what their experiences were with the syndrome.

Not in Control

The second theme that emerged from the coding process was not in control. Participants expressed the feeling of not being in control or having little control over the symptoms associated with PCOS including hirsutism, irregular periods, and, especially, weight gain. The feeling of not being in control was likely substantiated by the feeling of 
not knowing and vice versa. Interestingly, while a few participants said medications had helped them to feel more in control of their symptoms, many expressed a total lack of control despite being under the care of a specialized health care provider for the management of PCOS. The theme was constituted by the two subthemes of weight concerns and taking the medicines. During interviews, participants often discussed feeling completely out of control when it came to their weight. Statements about lack of control were often followed with comments about how taking the medicines was all they could do even if they did not know exactly why they were taking the medication or how it was benefiting them.

One participant expressed her lack of control and frustration regarding her weight saying, "Every time I try to lose weight, I'll lose like five or ten pounds, then I gain twenty back." Another said weight gain was the most stressful symptom she experienced and described her experience stating, "[The weight gain] is so hard...just recently l've gained a lot more...definitely I feel like I have very little control." Regarding the second subtheme, when participants were discussing their sense of control over symptoms, having to take medicine was often mentioned. One participant expressed her the challenges she faced with control and medications:

My control over irregular periods is like a zero. I can't control it at all. Every now and then my birth control will work on it...but most of the time it doesn't... With the hair growth, I have no control. Um, it's one of those things that I'm having to see doctors about [sighs]. 
Another described her experience with irregular periods and facial hair:

I don't really have any control over it. I just have to take my medicine. [The doctor] said it was supposed to clear up after a while... but, I don't feel like I have any control over it. It's just something 1 have to deal with.

\section{Not Now, Not Ever}

The most prevalent concern among the participants surrounded uncertainty regarding menstruation and fertility. The theme not now, not ever, represents a common question either indirectly or directly expressed by participants wondering about their menstrual cycle and the implications of irregularity in the future. Thus, this theme included two subthemes: irregular periods and fertility concerns. Participants were concerned about not having their periods currently and were stressed due to not knowing what to expect and not having control over their cycles. Consequently, the concern over not having regular periods now led participants to question whether they would ever have normal periods and if they would ever be able to have children. This theme and both subthemes are supported in this statement made by one of the participants when discussing how stressful it was having irregular periods:

I'd always worry about things, like, I worry about anything and everything...like, I hadn't been having sex, but I was like, "Oh my goodness, what if I'm pregnant," or like, "What if I'm never gonna be able to have a kid," stuff like that... because I didn't have my period, I was just like, "I don't know."

Another participant expressed similar concerns, stating, “...they say if I don't start my periods and stuff I may not be able to have babies in the future." The concern over fertility was prominent, and quite anxiety provoking for many of the participants. Regarding the threat of future infertility, one participant said, "That is something that 
actually, you know, scares me because I want children, but, you know, there's that great possibility that I can never have them." Although the majority of the participants had only been diagnosed with PCOS for only a year or less, the threat of having trouble conceiving in the future seemed to be something many of the girls had thought a great deal about. For example, one participant said, "I guess that [is] just something you always kind of worry about, but l've kind of accepted the fact that if I can't get pregnant...I can adopt." Others also mentioned plans to adopt, and another mentioned surrogacy as an option if she could not conceive in the future.

Not Horrible, Not the Best

While the other three themes represented negative aspects of PCOS experienced by the participants, the coding process revealed a final theme representing a more content and neutral point of view from the participants regarding PCOS. The theme not horrible, not the best emerged as an overall conclusion for the participants as they described their individual experiences with PCOS. As much as they disliked the symptoms, felt out of control, stressed, or uncertain, the general consensus was that living with PCOS certainly was not the worst situation they could think of. The two subthemes included in this theme were body image and managing symptoms as these were the two main issues described as being not horrible, but not the best by participants. Illustrating the first subtheme, one participant said, "With acne and weight gain, it's really a struggle with my self-esteem...but it's not like super horrible, but it's not great either." Illustrating the subtheme managing symptoms, another participant described her overall experience 
managing the symptoms of PCOS from mood swings to painful, irregular periods by stating, "It really hasn't been horrible...but it's not the best [chuckles]."

It is important to note the majority of the participants did not feel that having PCOS had negatively impacted any of their relationships. While there was repeated expression of concern over symptoms impacting outward appearance such as weight and facial hair growth, none of the participants expressed concern over or difficulty with dating or feeling unattractive to others. A few participants talked about their boyfriends, but did not express any negative impact from having PCOS on these relationships. Also, while many felt negatively toward taking medicine, there was a general sense of resolve among the participants when it came to the management of PCOS. Despite lack of knowledge and control, the participants found ways to cope with PCOS by talking to their mothers, doing hobbies, and thinking about things other than their experiences with PCOS.

Research question7- To what extent do the quantitative results about stressors, perceived control, coping, and depression converge with the interview data reporting the coping experiences of adolescent girls with PCOS?

Following the analysis of both the quantitative and qualitative results, the data were merged and assessed to determine if and how the findings from the two datasets were congruent or divergent (Creswell \& Plano Clark, 2011). Stress, lack of control, and maladaptive coping were theorized to be associated with depression. Due to the limited sample size, definitive conclusions were difficult to make considering the quantitative 
results. However, the qualitative findings, do lend support to the quantitative results and thus, converge to provide a greater understanding of the experiences adolescent girls have with PCOS.

First, girls reported feeling moderately high levels of stress and minimal to moderate levels of control over symptoms associated with PCOS. Irregular periods, and the threat of future infertility were found to be among the greatest stressors in the quantitative data analysis. Also, girls reported little control over irregular periods and the threat of future infertility. Supporting these findings was the theme that evolved from the qualitative data, not now, not ever, concerning the uncertainty over not having a regular period as an adolescent and pondering the potential implications of irregularity on future fertility.

The sample of adolescents who participated in this study were overall very depressed. Lower perceptions of control was a significant predictor of greater depression in the multiple linear regression analysis. Themes from the qualitative data supporting these quantitative findings were the themes not knowing and not in control. The girls expressed feelings of being out of control, not knowing what was going on with their health, and sometimes ambivalent about their health. They also expressed feeling different from others.

Girls' perceptions of their mothers' feelings related to PCOS were difficult to predict. Perception of maternal concern was not found to be a predictor of depression. Acceptance was the coping behavior used to the greatest extent by the participants. As 
girls perceived their mothers to be more concerned about their symptoms, girls increased their use of acceptance. The majority of girls described being able to talk to their mothers about PCOS and, in some cases, felt they could relate more to their mothers having experienced symptoms associated with PCOS, especially if the mother had experienced female reproductive issues. The qualitative theme relating to these findings might be not horrible, not the best. PCOS symptoms were stressful and girls felt little control, but they found ways to cope with the symptoms. Perhaps having a mother to confide in and to go to for help with managing the symptoms of PCOS assisted girls in coping.

\section{Summary}

The findings from the data analysis of both the quantitative and qualitative research findings were presented in this chapter. Additionally, the psychometric properties of each data collection instrument were reported and the characteristics of the sample were described. The chapter was concluded with a discussion on how the quantitative and qualitative data converged to answer the mixed methods research question. 


\section{CHAPTER 5}

\section{DISCUSSION AND RECOMMENDATIONS}

This chapter discusses the results, limitations, and recommendations for practice and future research. There existed a need for this mixed methods study due to a lack of existing literature surrounding psychosocial disorders, namely depression, among adolescent girls with PCOS and the ways in which they cope with their diagnosis and symptoms (Anderson, 2006; Dorkas et al., 2011; Dowdy, 2012; Kerchner et al., 2009). The purpose of this study was to explore relationships among PCOS-related stressors, coping, and depression among adolescent girls with PCOS. Research questions addressed which aspects of PCOS girls found most stressful and least controllable. Perceptions of maternal stress related to PCOS were examined as well as coping behaviors used by adolescent girls with PCOS. Stressors, controllability, and coping behaviors as predictors of depression were explored. Finally, interviews provided insight into the lived experiences of girls with PCOS. According to the convergent parallel mixed methods design, quantitative and qualitative data were converged to provide a more robust understanding of adolescent girls' experiences with PCOS (Creswell \& Plano Clark, 2011). 


\section{Discussion of Findings}

Lazarus and Folkman (1984) identified coping as a key concept for research involving health and adaptation to chronic illness, whereas one's appraisal of stress and perceived control over the stressor could impact one's ability to cope. Primary and secondary appraisals involve, first, determining the nature of the stressor and, second, determining what can be done about the stressor. Thus, control becomes an important variable in the coping process. In most cases, when greater control is perceived, overall stress is decreased (Lazarus \& Folkman, 1984). One's sense of control may impact one's use of coping resources and may determine whether maladaptive or adaptive coping strategies are implemented (Lazarus \& Folkman, 1984). In this current study, adolescent girls' perceptions of stress and control over aspects of PCOS, along with their perceptions of maternal stress, were explored using the researcher-developed instrument, PCOS Stressor Scale (PCOSSS). Despite challenges with instrumentation, it was evident that girls were concerned about the aspects of PCOS. The qualitative data supported that girls were concerned as they expressed their frustrations and worry about PCOS. Participants in this study appraised the aspects of PCOS to be stressful and threatening to their health, self-image, social functioning, and future plans. These findings are consistent with existing literature on girls and women with PCOS (Dowdy, 2012; Himelein \& Thatcher, 2006; Jones et al., 2011).

Girls' Concerns and Perceived Maternal Concerns

The current study revealed girls perceived their mothers to be concerned about the same aspects of PCOS that they were concerned about including weight, irregular 
periods, and the threat of future infertility. Girls reported having more control over acne and hirsutism, and did not think their mothers were concerned about these symptoms. The interview data revealed that girls needed and appreciated maternal support. Many participants reported talking to only their mothers about symptoms of PCOS and feeling closer to their mothers because their mothers had experienced similar symptoms or other female reproductive issues. Similarly, Wiebe et al. (2005) studied adolescents with type 1 diabetes and identified greater maternal involvement and support to be predictor for more favorable health outcomes in adolescents. Wiebe et al. (2005) discussed the implications of how adolescents perceive maternal involvement whether it be controlling, uninvolved, or collaborative. As adolescence is a stage of life involving the development of autonomy in decision making and responsibility for one's health, perceiving a parent to be uninvolved or controlling can be detrimental to health outcomes. Wiebe et al. (2005) found adolescent girls who appraised their mothers as controlling had reduced quality of life compared to those who appraised their mothers to be collaborative.

Overall, adolescents who appraised their mothers to be collaborative had better adherence and metabolic control. The researchers suggested appraised collaboration may promote an adolescent's self-efficacy and may help to regulate negative emotions.

While the current study did not examine whether girls perceived their mothers to be uninvolved, controlling, or collaborative, the results demonstrated a shared concern over PCOS between mother and daughter. Interviews with the participants revealed the girls found comfort in the support their mothers provided while managing the aspects of PCOS. Further discussion will illuminate how adolescent girls' perceptions of maternal 
concern impacted their coping behaviors. Before addressing coping and depression among the participants, the research question exploring adolescent girls' perceptions of control will be discussed.

Girls' Perceived Control

Controllability has been identified as a crucial determinant of coping efficacy (Compas, Jaser, Dunn, \& Rodriguez, 2012). Low perceived control over stress has been associated in related literature with less favorable psychological outcomes (Clarke, 2006; Compas et al. 1988; Lazarus \& Folkman, 1984; Zimmer-Gembeck \& Skinner, 2015). Exploring adolescent vulnerability and the distress of peer rejection, Zimmer-Gembeck and Skinner (2015) found a positive correlation between depressive symptoms and less perceived control among adolescents. Additionally, they found less perceived control to also be associated with the use of less adaptive coping behaviors, such as distraction. The current study revealed girls perceived very little control over the aspects of PCOS. Lower control was found to be a predictor of greater depression among the participants and perceptions of lack of control were evident and were supported by the qualitative data.

It is interesting that girls perceived little to no control over their symptoms, namely irregular periods, despite being on medications to control periods and the hyperandrogenic effects of PCOS. Moreover, the girls were being seen on a regular basis by specialized health care providers for the management of PCOS, which likely provided them with ample opportunities to discuss their concerns regarding PCOS. These findings could suggest a lack of effective communication between health care providers and 
patients regarding the disease process and treatment plans; however, it could also be the result of complex relationships between girls' perceptions of PCOS and ways of coping. For example, one participant explained during the interview that she was concerned about PCOS and was uncertain about many aspects of the disease including future infertility, however, she was content to take the medications as her health care provider prescribed and did not want to discuss her concerns. She expounded that she was not interested in learning more about PCOS, and if she had any questions about it later in life, she would ask her health care provider then.

Acceptance and self-distraction were the most frequently used coping behaviors among the participants. Perhaps these coping behaviors contributed to a general perception of low control. The qualitative data revealed that participants, in general, did not fully understand PCOS. If they were content to take prescribed medications without asking questions and discussing concerns with health care providers, this could have perpetuated a feeling of having little to no control secondary to a lack of knowledge about one's health. On the other hand, it is possible girls could be intimidated by the complexity of PCOS when discussing the disease with a provider, leading to feelings of low control, which could contribute to the use of acceptance or distraction as a coping behavior.

Further research is necessary to determine the connection between girls' perceived control over aspects of PCOS, coping behaviors, and relationships with health care providers. To further explore concern and control, mirroring the study conducted by Wiebe et al. (2005), future studies could explore whether girls perceive their health care 
providers to be uninvolved, controlling, or collaborative when addressing their concerns related to PCOS. It would be interesting to determine how open patient-provider communication and problem-solving between patients and providers could impact the perception of control over PCOS related stressors among adolescent girls with PCOS.

\section{Coping}

The current study did not reveal any negative impact on coping or depression based on perceptions of maternal concern over the aspects of PCOS. There were no significant increases in use of maladaptive coping behaviors such as substance use or behavioral disengagement when perceptions of maternal concern increased. In fact, as the perception of maternal stress increased, the use of acceptance increased. Acceptance has been identified as a secondary-control coping strategy (Compas et al., 2012). With secondary-control coping an individual uses behaviors such as acceptance or distraction in effort to adapt to stress (Compas et al., 2012). As stated previously, acceptance and self-distraction were types of coping behaviors reported as being used most frequently by the participants. These forms of coping are considered healthy and adaptive (Morling \& Evered, 2006). Like Wiebe et al. (2005) found, mothers who collaborate with their adolescent, engaging in discussions and problem-solving assist their adolescent to more effectively cope with health-related stressors. This was an encouraging finding for mothers helping their daughters to navigate the management of PCOS.

Secondary-control coping is comprised of two actions including adjusting oneself and accepting the environment (Morling \& Evered, 2006). Several studies examining coping in adolescents with chronic illness or pain have demonstrated the use of 
secondary-control coping to be associated with lower depression and anxiety (Compas et al., 2006; Edgar \& Skinner, 2003; Miller et al., 2009; Thomsen et al., 2002).

Morling and Evered (2006) argued that the motive of secondary-control coping is to fitin, and does not serve as a motive to control, which is the purpose of primary-control coping. Primary-control coping serves the basic human need for control through mastery-oriented measures, whereas, secondary-control coping serves to achieve measures of well-being, relatedness, serenity, and consistency. Primary-control coping behaviors may be grouped into categories of coping behaviors including active coping or problem-based coping. Secondary-control coping behaviors could be labeled as passive or emotion-focused. The important point to remember about coping behaviors is that regardless of how ways of coping are labeled, one cannot assume all active or problembased types are adaptive and all passive or emotion-focused coping behaviors are maladaptive. All types of coping may have multiple functions (Lazarus, 1996). Therefore, Morling \& Evered (2006) argued secondary-control coping may not be functionally secondary to primary-control coping at all, but rather a unique way of coping that may serve different purposes depending on the stressor.

Compas et al. (1988) examined the relationship between perceived control, coping behaviors, and reported stress. According to the findings, when adolescents matched perceived low control with emotion-focused coping strategies they reported lower stress. Additionally, when adolescents matched situations where they perceived greater control with problem-focused coping strategies they reported lower stress. Lazarus and Folkman (1984) defined problem-focused coping as being aimed at directly managing or altering 
the given stressor. Emotion-focused coping was defined as behaviors directed at regulating emotional responses to the stressor (Lazarus \& Folkman, 1984).

Overall, girls more commonly use emotion-focused coping strategies when compared to their male counterparts (Calvete, Camara, Estevez, \& Villardón, 2011). The girls in the current study reported using emotion-focused coping most frequently to cope with PCOS stressors. Considering participants in the current study used emotion-focused coping behaviors more frequently than problem-focused coping behaviors in response to a stressor associated with little to no perceived control, it is interesting that participants still reported moderately high levels of stress and concern over the aspects of PCOS. Going back to the assumption that low control contribute to greater stress, perhaps girls did not report feeling less stress because they still perceived little control over the stressors (Lazarus and Folkman, 1984). The girls chose to cope mostly through acceptance and self-distraction, and while these secondary-control coping behaviors can be considered adaptive, the fact that the motive of secondary-control coping is to fit-in rather than to increase control over stress, may have contributed to the high depression scores reported by the participants. Further discussion will illuminate findings examining coping and depression among the adolescent girls with PCOS in the current study.

A few other areas for consideration in future research regarding coping in adolescent girls with PCOS surround issues coping behaviors used in relation the timing of diagnosis and personality. Aldridge and Roesch (2007) examined coping in children with cancer and described associations between types of coping behaviors with overall adjustment at varying intervals after diagnosis. For example, at six months to one year 
after diagnosis with cancer, children who used problem-focused or approach coping behaviors demonstrated poorer adjustment. However, four to five years after diagnosis these types of coping were associated with better adjustment. Future research could be done in this area to investigate how coping behaviors predict depression or psychosocial well-being over time, as the adolescent develops cognitively and as time passes from the initial time of diagnosis.

The influence of personality on coping is not fully understood due to difficulties measuring personality and coping, overreliance on cross-sectional studies, inadequate control over variables, and overall lack of attention to interactions between personality traits and coping strategies (Carver \& Conner-Smith, 2010). Studies have found adolescent girls to report more depressive symptoms, more perceived stress, and less perceived control than their male counterparts when encountered with similar types and amounts of stress (Calvete et al., 2011; Zimmer-Gembeck \& Skinner, 2015). Therefore, Zimmer-Gembeck and Skinner (2015) concluded rather than gender differences explaining coping and health outcomes, the way an adolescent reacts emotionally and appraises a stressor provides a more direct explanation of coping strategies and outcomes. Personality influences coping behaviors in many ways including the individuals biological view of stress, the likelihood of experiencing greater or less stress resulting from personality type, responses to stress, coping strategies, and effectiveness of coping in relation to one's personality and perceptions (Carver \& Conner-Smith, 2010; Compas et al., 2012). Addressing personality in relationship to coping and depression among adolescent girls would be helpful in explaining depression scores and would provide 
valuable information for health care professionals seeking to provide more individualized care and counseling to girls coping with PCOS.

Depression

The current study revealed high levels of depression reported by participants on the PHQ-9. While girls discussed feeling out of control and concerned about PCOS stressors, they did not discuss feeling depressed during the interviews. On the contrary, most described ways of coping, including confiding in their mothers about their concerns, doing other activities to keep their minds off the symptoms of PCOS. Overall, the coping behaviors participants reported using most frequently were adaptive and were not predictors of greater depression.

'These findings led to a question of why girls' depression scores were so elevated. While the PHQ-9 is a valid and reliable tool, commonly used to measure adolescent depression, Richardson et al. (2010) did report when used in the adolescent population the PHQ-9 had a higher sensitivity and a lower specificity compared to use in adult populations. Nevertheless, the PHQ-9 had a high sensitivity (89.5\%) and a good specificity $(\mathbf{7 8 . 8 \%})$ for detecting major depression among adolescents (Richardson et al., 2010). Richardson et al. (2010) predicted the higher false-positive rate in the adolescent population may be a result of a high rate of subthreshold symptoms, adjustment disorders, and/or overlap with other mental health disorders. While there may be a slightly higher rate of false-positive results when using the PHQ-9 for adolescents, the instrument is highly sensitive, making it an excellent screening tool, as it is unlikely to miss an adolescent with depression. Kroenke et al. (2001) recommended all responses on 
the PHQ-9 should be verified by a clinician and a definitive diagnosis should be made only when the clinician takes into account how well the person understood the questionnaire. It is also suggested that severity of depression be monitored over time for newly diagnosed individuals or those being treated for depression by repeating the PHQ9 at regular intervals. While the design of the current study did not allow the researcher to verify answers on the PHQ-9 with adolescents, and the questionnaire was only given once, the depression scores are still valuable and indicate girls were depressed, regardless of severity.

The current study revealed girls' perception of control over PCOS-related stressors to be a significant predictor of depression. Greater perceived control was associated with lower depression. Therefore, the findings from this study are consistent with other research indicating low perceived control is associated with negative health outcomes (Calvete et al., 2011; Compas et al., 2012; Lazarus \& Folkman, 1984; ZimmerGembeck \& Skinner, 2015). While perceived control did predict depression, the girls did not discuss feeling depressed during the interviews. Considering the prevalence and degree of reported depression on the PHQ-9, the researcher assumed a theme of depression would have emerged from the interview data. Instead, the theme of PCOS being described by participants as not horrible, not the best emerged. Girls did not express feeling isolated from peers or disgusted with their appearance. Perhaps this is because the outwardly observable symptoms of PCOS, with the exception of weight, such as hirsutism and acne, were perceived as the least stressful and most controllable aspects of PCOS. Interviews with the participants did not reveal that dealing with aspects of 
PCOS limited girls' activities or impacted their relationships negatively. The negative impact of PCOS directed inwardly, with girls expressing feeling like they did not fully understand the illness, felt out of control, and worried about the future. Perhaps girls underestimated or did not realize the impact their perception of having low control had on their depressive symptoms and did not discuss this correlation during the interviews. A question directly related to depression during the interviews would have been helpful in exploring this phenomenon. Another important consideration to account for the discrepancy between quantitative and qualitative findings related to depression is that there could have been factors unrelated to PCOS contributing to depression in the participants. Future research examining this population could explore all coexisting stressors and determine how PCOS stressors compare to other stress in predicting depression.

Still, many other studies examining depression among adolescent girls with chronic illness have produced similar results. In a study of adolescent girls with systemic lupus erythematous, Ji et al. (2012) found nearly all of the girls had increased depressive symptoms when compared to healthy controls. Appearance concern and older age predicted greater depression among the girls. Due to the high depressive symptoms and risk for developing depression, Ji et al. (2012) recommended adolescent girls with SLE be assessed routinely for depression. Pollock et a. (2011) conducted a study of depression in adolescent boys and girls with neck and shoulder pain. The association between depressed mood and neck and shoulder pain was significantly stronger for girls than boys. Pollock et al. (2011) did not evaluate perceived stress or control over the neck 
and shoulder pain; however, it would be interesting to know if girls perceived the same amount or type of pain as more stressful or less controllable than boys, therefore leading to a more depressed mood. In the study discussed previously by Calvete et al. (2011), girls scored higher on depressive symptom even though they reported having similar numbers of social stressors when compared to their male counterparts. The difference was that girls scored higher on the perceived severity of the stressors than boys, and thus had higher depression scores (Calvete et al., 2011). In an earlier study, Seiffge-Krenke (1995) argued that girls perceive the same stressors as up to four times more challenging and stressful than boys do.

Considering the findings from these studies involving adolescent girls with chronic illness or pain and comparing depression in girls and boys, it could be concluded that since girls are more likely to perceive higher amounts of stress from life stressors, they are already at risk for higher depression. Furthermore, since adolescence is a critical development time of life characterized by dramatic physical and psychological changes, any threats to the adolescents' health which could affect self-perception, self-efficacy beliefs, or peer and family relationships could be perceived as stressful, and even more so for girls who perceive stressors to be more severe (Calvete et al., 2011; Compas, 1987; Pirgon, Sandal, Gökçen, Bilgin, \& Dündar, 2015; Seiffge-Krenke, 1995; Trent et al., 2002). Although other unmeasured variables could have impacted depression scores, the results from the current study certainly support a high prevalence of depression in adolescent girls with PCOS, with lower perceived control over PCOS-related stressors predicting greater depression. 


\section{Recommendations for Nursing Practice}

The results of this study described perceptions of concern, control, maternal concern, ways of coping, and depression in adolescent girls with PCOS. Girls were concerned about PCOS and perceived very little control over the disease which was a predictor of depression among the girls. Girls who participated in this study were being seen regularly by health care providers for the treatment of PCOS. Despite seeing a health care provider at frequent intervals, sometimes as often as every two weeks, the girls did not know much about PCOS, had many concerns, perceived little control, and were very depressed.

Emerging from the findings of this study are recommendations for nurses and other health care professionals providing care to adolescent girls with PCOS. First, health care providers must understand developmental stages in order to best care for adolescents and must learn to actively engage adolescents in conversation about their health. Adolescents are more likely to open up to health care providers when the conversation focuses on them as a person rather than their health problems. Health care providers must be patient and willing to talk to adolescents about things they are interested in such as friends and extracurricular activities in order to establish rapport and begin important discussions about physical and psychosocial health (Hogan-Quigley, Palm, \& Bickley, 2012). If providers do not invest time in developing relationships with patients, they will likely miss important concerns from the adolescent, as adolescents are often reluctant to ask questions (Hogan-Quigley et al., 2012). Without having frank discussions about concerns girls are having related to PCOS, providers will not be able to 
address those concerns. This will perpetuate a perception of low control in girls with PCOS and, in turn, will perpetuate the risk for depression in this population. Girls need to understand the disease process more thoroughly, and they need to understand the purpose of medications and what they can do to best care for themselves in the present and how those actions can impact their future self. Open lines of communication between health-care providers and patients which serve to enhance girls' knowledge, understanding, and sense of support are critical in addressing girls concerns and perceptions of control over aspects of PCOS (Dowdy, 2012).

Second, this study addressed girls' perception of maternal stress related to the aspects of PCOS. Encouragingly, girls felt their mothers shared their concerns about PCOS, and described their mothers to be an important support person. Girls did not perceive their mothers to be concerned about their physical appearance, and girls' perceptions of maternal concern was not related to greater use of maladaptive coping behaviors. Thus, the second recommendation for nurses caring for adolescents with PCOS is to involve the patient's mother or guardian as much as possible in the plan of care. While it is imperative to explain and maintain provider-patient confidentiality, the provider's goal should be to help adolescents bring their concerns and questions regarding what they are experiencing with PCOS to their mothers (Hogan-Quigley et al., 2012). Wiebe et al. (2005) found adolescents who perceived their mothers to be a collaborative partner in the management of diabetes had better health outcomes. Collaboration, consisting of discussion, joint-decision making, negotiation, and problemsolving should be the goal of the mother-daughter relationship (Wiebe et al., 2005). 
Educating girls and their mothers about the importance of a collaborative relationship, and fostering that relationship through open dialogue with mother, daughter, and health care provider during office visits represents a step toward providing more effective, evidence-based care for this population.

Finally, it was apparent from the current study that girls with PCOS are at risk for depression. Other studies involving adolescent girls with chronic health problems have also detected high depressive symptoms (Ji et al., 2012; Pollock et al., 2011). Nurses and other health care providers caring for girls with PCOS should be aware of this risk and should routinely screen their patients for depression. Mothers or guardians, as the collaborative partner, should be aware of the risk for depression and encouraged to keep channels of communication open with her daughter. Health care providers should educate girls and their mothers on signs and symptoms of depression, and should encourage girls and/or mothers to communicate any concerns related to depression to the provider promptly.

\section{Recommendations for Future Research}

The current study focused on how stress and control impacted coping and depression in adolescents with PCOS in effort to address a gap in the literature (Anderson, 2006; Dorkas et al., 2011; Dowdy, 2012; Kerchner et al.,2009; Rofey et al., 2009; Weiss \& Bulmer, 2011). Additionally, this study represented the first step toward exploring the impact on mother-daughter relationships on coping in this population. The literature on providing appropriate psychosocial care to adolescents with PCOS is scarce. The findings of the current study point to a need for future research to examine how girls' 
perceptions of their relationships with their mothers and health care providers relate to perceived control and ways of coping. Studies examining these relationships would yield further insights into developing more effective care for girls with PCOS through communication, education, and support. To further explore the value of relationships and communication, future research could involve developing and implementing an educational intervention for girls with PCOS and their mothers or guardians in which the physiology, pathophysiology, and typical management of PCOS were addressed along with an interactive discussion about common signs, symptoms, and concerns. Issues around control of PCOS during adolescence and in the long-term could be addressed within the dyad. Perceived stress, control, coping, and depression between girls having attended the course and those who did not could be compared to determine if this important information could improve short-term and long-term outcomes. Several other variables and relationships could be explored through the implementation of this type of educational and supportive intervention.

Additionally, future longitudinal studies are needed to examine control and coping in adolescent girls over time to more explicitly understand needs of the adolescent early after diagnosis versus needs of those having had PCOS over a longer length of time. Perceptions of stress, control, and ways of coping would also be interesting to examine related to age to determine how the adolescent adapts at different ages over time. Findings from these future studies would allow health care providers to better provide more individualized care, in effort to increase quality of life among this population and decrease negative health outcomes. 


\section{Limitations of the Study}

The most substantial limitation of this study was the limited quantitative sample size due to unforeseen challenges with recruitment. This resulted in a study that was likely underpowered to detect statistically significant relationships among variables. In addition, estimates of correlations, beta weights, and $\mathrm{R}^{2}$ should be viewed with caution.

The PCOSSS was a researcher developed scale and was used for the first time in this study. As with any newly developed instrument, there is the potential for weak psychometrics and in this case, the internal consistency reliability of the girls' concerns subscale was unacceptable. The decision was made to examine each item of the scale individually. Because of this, the researcher was unable to estimate a total score for concerns, which may have been a better predictor of depression. Additional psychometric work on the PCOSSS is warranted.

The cross sectional design limited the study findings as the data were gathered one time and effects over time were not studied, particularly in regard to depression scores among participants. It is possible that the relationship between control and depression is bi-directional such that increased depression may lead to lower perceptions of control of PCOS stressors. Longitudinal studies of girls with PCOS would help clarify these relationships.

Conducting the study at one health care system where many of the participants were cared for by the same health care providers also limited the generalizability of the results outside the population of the health care system. Further research with larger sample sizes, utilizing multiple sites, and conducted over time would provide greater 
insight into variables impacting stress, control, coping, and depression among adolescent girls with PCOS.

\section{Conclusions}

This study sought to explore coping and depression among adolescents, along with their perceptions of stress, control, and maternal stress. The findings of this study contribute to the developing body of research surrounding PCOS in adolescents by providing a description of adolescent girls' experiences with PCOS and how those experiences support and explain reported perceptions of concern, control, and depression. As perceived low control predicted depression among the participants, recommendations were made for nursing practice and future research to pursue the goal of improving girls' perceptions of control over PCOS related stressors. Findings from this study suggest the best strategies for increasing girls' perceived control over stressors is to foster more constructive, collaborative relationships between girls, their mothers, and health care providers. This study provided another step toward a better understanding of the experiences of adolescent girls with PCOS. Ultimately, through empowering girls with knowledge and understanding of their bodies and the implications of PCOS, and by building and fostering collaborative relationships where trust prevails and continual, open discussion about girls' concerns is ensured, health care providers can positively impact the overall psychosocial well-being of their patients and decrease depression among this population. 


\section{References}

Adali, E., Yilizhan, R., Kurdoglu, M., Kolusari, A., Edrine, T., \& Sahin, H. (2008). The relationship between clinic-biochemical characteristics and psychiatric distress in young women with polycystic ovary syndrome. Journal of Internal Medicine Research, 36, 1188-1196. doi: 10.1177/147323000803600604

Agapova, S. E., Cameo, T., Sopher, A. B., \& Oberfield, S. E. (2014). Diagnosis and challenges of polycystic ovary syndrome in adolescence. Seminars in Reproductive Medicine, 32, 194-201. doi: 10.1055/s-0034-1371091

Aldridge, A. A. \& Roesch, S. C. (2007). Coping and adjustment in children with cancer: A meta-analytic study. Journal of Behavioral Medicine, 30, 115-129. doi:10.1007/s10865-006-9087-y

Anderson, C. L. (2006). Hyperandrogenic disorders. In K. D. Schuiling \& F. E. Likis (Eds.), Women's gynecologic health (pp. 533-559). Sudbury, MA: Jones and Bartlett.

Bannon, W. M. (2013). The 7 steps of data analysis: A manual for conducting a quantitative research study. Brooklyn, NY: Stats Whisperer Press.

Beauchamp, T. L. \& Childress, J. F. (2013). Principles of biomedical ethics. New York, NY: Oxford University Press.

Benson, S., Hahn, S., Tan, S., Janssen, O. E., Schedlowski, M., \& Elsenbruch, S. (2010). Maladaptive coping with illness in women with polycystic ovary syndrome. Journal of Obstetric, Gynecologic, \& Neonatal Nursing, 39, 37-45. doi:10.1111/j.1552-6909.2009.01.086x

Bonny, A. E. \& Gomez-Lobo, V. (2016, April). The diagnosis of PCOS in adolescence [PDF document]. Presentation given at the North American Society for Pediatric and Adolescent Gynecology $30^{\text {th }}$ Annual Clinical and Research Meeting, Vancouver, Canada.

Bonny, A. E. \& Javed, A. (2016, April). The honest hairy truth about PCOS: What your patient wants to know but is afraid to ask [PDF document]. Presentation given at the North American Society for Pediatric and Adolescent Gynecology $30^{\text {th }}$ Annual Clinical and Research Meeting, Vancouver, Canada 
Brytek-Matera, A. (2007). Anorexia nervosa among French adolescent females in relation to self-esteem, coping strategies, anger expression, and anger control. Archives of Psychiatry and Psychotherapy, 4, 53-57. Retrieved from http://www.kom-red-wyd-ptp.com.pl/indexen.php

Calvete, E., Camara, M., Estevez, A., \& Villardón, L. (2011). The role of coping with social stressors in the development of depressive symptoms: Gender differences. Anxiety, Stress, \& Coping, 24, 387-406. doi:10.1080/10615806.2010.515982

Carver, C. S. (1997). You want to measure coping but your protocol's too long: Consider the Brief COPE. International Journal of Behavioral Medicine, 4, 92-100. Retrieved from http://www.springer.com

Carver, C. S., \& Connor-Smith, J. (2010). Personality and coping. Annual Review of Psychology, 61, 679-704. doi:10.1146/annurev.psych.093008.100352

Carver, C. S., Scheier, M. F., \& Weintraub, J. K. (1989). Assessing coping strategies: A theoretically based approach. Journal of Personality and Social Psychology, 56, 267-283. Retrieved from http://www.apa.org

Clarke, A. T. (2006). Coping with interpersonal stress and psychological health among children and adolescents: A meta-analysis. Journal of Youth and Adolescence, 35 , 11-24. doi:10.1007/s10964-005-9001-x

Clayton, W. J., Lipton, M., Elford, J., Rustin, M., \& Sherr, L. (2005). A randomized controlled trial of laser treatment among hirsute women with polycystic ovary syndrome. British Journal of Dermatology, 152, 986-992.

doi:10.1111/j.1365.2133.2005.06426.x

Collier, M. J. \& Rieder, J. (2008). Detecting polycystic ovarian syndrome in teens. Contemporary $O B / G Y N, 53,44-49$. Retrieved from http://www.advanstar.com/index\%5Fallpubs.html

Compas, B. E. (1987). Coping with stress during childhood and adolescence. Psychological Bulletin, 101, 393-403.

Compas, B. E., Boyer, M. C., Stranger, C., Colletti, R. B., Thomsen, A. H., Dufton, L. M., \& Cole, D. A. (2006). Latent variable analysis of coping, anxiety/depression, and somatic symptoms in adolescents with chronic pain. Journal of Consulting and Clinical Psychology, 74, 1132-1142. doi:10.1037/0022-006X.74.6.1132 
Compas, B. E., Jaser, S. S., Dunn, M. J., \& Rodriguez, E. M. (2012). Coping with chronic illness in childhood and adolescence. Annual Review of Clinical Psychology, 8, 455-480. doi:10.1146/annurev-clinpsy-032511-143108

Compas, B. E., Malcarne, V. L., \& Fondacaro, K. M. (1988). Coping with stressful events in older children and young adolescents. Journal of Consulting and Clinical Psychology, 56, 405-411.

Creswell, J. W. \& Plano Clark, V. L. (2011). Designing and conducting mixed methods research ( $2^{\text {nd }}$ ed.). Thousand Oaks, CA: Sage.

Davis, S. R. \& Tran, J. (2001). Testosterone influences libido and well being in women. Trends in Endocrinology and Metabolism, 12, 33-37. doi:10.1016/S10432760(00)00333-7

Dorkas, A., Clifton, S., Futterweit, W., \& Wild, R. (2011). Increased risk for abnormal depression scores in women with polycystic ovary syndrome. Obstetrics \& Gynecology, 117, 145-152. doi:10.1097/AOG.0b013e318202b0a4

Dowdy, D. (2012). Emotional needs of teens with polycystic ovary syndrome. Journal of Pediatric Nursing, 27, 55-64. doi:10.1016/j.pedn.2010.08.001

Dramusic, V., Rajan, U., Chan, P., Ratman, S., \& Wong, E. (1997). Adolescent polycystic ovary syndrome. Annals of the New York Academy of Sciences, 816, 194-208. doi:10.1111/j.1749-6632.1997.tb52143.x

Edgar, K. A. \& Skinner, T. C. (2003). Illness representations and coping as predictors of emotional well-being in adolescents with type 1 diabetes. Journal of Pediatric Psychology, 28, 485-493. doi:10.1093/jpepsy/jsg039

Eisenhardt, S., Schwarzmann, N., Henschel, V., Germeyer, A., von Wolff, M., Hamann, A., \& Strowitzki, T. (2006). Early effects of metformin in women with polycystic ovary syndrome: A prospective randomized, double-blind, placebo-controlled trial. Journal of Clinical Endocrinology and Metabolism, 91, 946-952. doi: $10.1210 /$ jc. $2005-1994$

Faucher, M. A. \& Schuiling, K. D. (2006). Normal and abnormal uterine bleeding. In K. D. Schuiling \& F. E. Likis (Eds.), Women's gynecologic health (pp. 507-532). Sudbury, MA: Jones and Bartlett. 
Ferriman, D. \& Gallwey, J. D. (1961). Clinical assessment of body hair growth in women. Journal of Clinical Endocrinology and Metabolism, 21, 1440-1447. doi:10.1210/jcem-21-11-1440

Folkman, S. \& Moskowitz, J. T. (2004). Coping: Pitfalls and promise. Annual Review of Psychology, 55, 745-774. doi:10.1146/annurev.psych.55.090902.141456

Ghazeeri, G., Fakih, A., Abbas, H. A., Harajly, S., \& Awwad, J. (2013). Anxiety, cognitive, and depressive assessment in adolescents with polycystic ovarian syndrome: A pilot study. Journal of Pediatric and Adolescent Gynecology, 26, 269-273. doi:10.1016.j.jpag.2013.04.005

Hahn, S., Janssen, O. E., Tan, S., Pleger, K., Mann, K., Schedlowski, M.,... Elsenbruch, S. (2005). Clinical and psychological correlates of quality-of-life in polycystic ovary syndrome. European Journal of Endocrinology, 153, 853-860. doi:10.1530/eje.1.02024

Hansen, K. A. (2014). What is new in polycystic ovary syndrome? Obstetrics and Gynecology, 124, 630-632. doi:10.1097/AOG.0000000000000433

Harris-Glocker, M., Davidson, K., Kochman, L., Guzick, D., \& Hoeger, K. (2010). Improvement in quality-of-life questionnaire measures in obese adolescent females with polycystic ovary syndrome treated with lifestyle changes and oral contraceptives, with or without metformin. Fertility and Sterility, 93, 1016-1019. doi:10.1016/j.fertstert.2009.08.006

Himelein, M. J. \& Thatcher, S. S. (2006). Depression and body image among women with polycystic ovary syndrome. Journal of Health Psychology, 11, 613-625. doi: $10.1177 / 1359105306065021$

Hogan-Quigley, B., Palm, M. L., Bickley, L. (2012). Bates' nursing guide to physical examination and history taking. Philadelphia, PA: Wolters Kluwer Health| Lippincott Williams \& Wilkins.

Hollinrake, E. M., Abreu, A., Sparks, A., Van Voorhis, B., \& Dorkas, A. (2005). Increased risk of depression in women with polycystic ovary syndrome. Fertility and Sterility, 84, S55. doi:http://dx.doi.org/10.1016/j.fertnstert.2005.07.131 
Ibanez, L., Valls, C., Potau, N., Marcos, M. V., \& de Zegher, F. (2000). Sensitization to insulin in adolescent girls to normalize hirsutism, hyperandrogenism, oligomenorrhea, dyslipidemia, and hyperinsulinism after precocious pubarche. Journal of Clinical Endocrinology and Metabolism, 85, 3526-3530. Retrieved from http://www.ncbi.nlm.nih.gov/pubmed/11061495

Izydorczyk, B. (2010). Body image among young females with anorexia nervosa and the structure of body image among their mothers. Archives of Psychiatry and Psychotherapy, 4, 61-67.

Ji, L., Jing, W., Yanyan, H., Min, W., Juan, X., \& Hongmei, S. (2012). Appearance concern and depression in adolescent girls with systemic lupus erythematous. Clinical Rheumatology, 31, 1671-1675. doi:10.1007/s10067-012-2071-8

Jones, G. L., Hall, J. M., Lashen, H. L., Balen, A. H., \& Ledger, W. L. (2011). Healthrelated quality of life among adolescents with polycystic ovary syndrome. Journal of Obstetric, Gynecologic, \& Neonatal Nursing, 40, 577-588. doi:10.1111/j.15526909.2011.01279.x

Karacan, E., Caglar, G. S., Gürsoy, A. Y, \& Yilmaz, M. B. (2014). Body satisfaction and eating attitudes among girls and young women with and without polycystic ovary syndrome. Journal of Pediatric and Adolescent Gynecology, 27, 72-77. doi:10.1016/j.jpag.2013.08.003

Kerchner, A., Lester, W. Stuart, S. P., \& Dorkas, A. (2009). Risk of depression and other mental health disorders in women with polycystic ovary syndrome: A longitudinal study. Fertility and Sterility, 91, 207-212. doi:10.1016/j.fertnstert.2007.11.022

Kiddy, D. S., Hamilton-Fairley, D., Bush, A., Short, F., Anyaoku, V., Reed, M. J., \& Franks, S. (1992). Improvement in endocrine and ovarian function during dietary treatment of obese women with polycystic ovary syndrome. Clinical Endocrinology, 36, 105-111. Retrieved from http://www.ncbi.nlm.nih.gov/pubmed/1559293

Kroenke, K., Spitzer, R. L., \& Williams, J. B. W. (2001). The PHQ-9: Validity of a brief depression severity measure. Journal of General Internal Medicine, 16, 606-613.

Krohne, H. W. (2001). Stress and coping theories. International Encyclopedia of the Social and Behavioral Sciences, 22, 15163-15170. doi:10.1016/B0-08-0430767/03817-1

Lazarus, R. S. (1993). Coping theory and research: Past, present, and future. Psychosomatic Medicine, 55, 234-247. 
Lazarus, R. S. (1996). The role of coping in the emotions and how coping changes over the life course. In C. Maletsta-Magni \& S. H. McFadden (Eds.), Handbook of emotion, adult development, and aging (pp. 289-306). New York, NY: Academic Press.

Lazarus, R. S. (1999). Stress and emotion: A new synthesis. New York, NY: Springer.

Lazarus, R. S. \& Folkman, S. (1984). Stress, appraisal, and coping. New York, NY: Springer.

Lee, H. \& Schepp, K. G. (2011). Ways of coping in adolescents with schizophrenia. Journal of Psychiatric and Mental Health Nursing, 18, 158-165. doi: $10.1111 / \mathrm{j} .1365-2850.2010 .01643 . \mathrm{x}$

Lincoln, Y. S. \& Guba, E. G. (1985). Naturalistic inquiry. Newbury Park, CA: Sage.

Maes, S., Leventhal, H., \& de Ridder, D. T. D. (1996). Coping with chronic diseases. In M. Zeidner \& N. S. Endler (Eds.), Handbook of coping: Theory, research, applications (pp. 221-251). New York, NY: John Wiley \& Sons, Inc.

McHaffie, H. E. (1992). Coping: An essential element of nursing. Journal of Advanced Nursing, 17, 933-940.

Miller, K. S., Vannatta, K., Compas, B. E., Vasey, M., McGordon, K. D., Salley, C. G., $\&$ Gerhardt, C. A., (2009). The role of coping and temperament in the adjustment of children with cancer. Journal of Pediatric Psychology, 34, 1135-1143. doi:10.1093/jpepsy/jsp037

Morling, B. \& Evered, S. (2006). Secondary control reviewed and defined. Psychological Bulletin, 132, 269-296. doi:10.1037/0033-2909.132.2.269

Neumark-Sztainer, D., Story, M. Hannan, P., Perry, C., \& Irving, L. (2002). Weightrelated concerns and behaviors among overweight and nonoverweight adolescents: Implications for preventing weight-related disorders. Archives of Pediatrics and Adolescent Medicine, 156, 171-178. doi:10.1001/archpedi.156.2.171

Nicandri, K. F. \& Hoeger, K. (2012). Diagnosis and treatment of polycystic ovarian syndrome in adolescents. Current Opinion in Endocrinology, Diabetes, and Obesity, 19, 497-504. doi:10.1097/MED.0b013e32835a1a03

Nunnally, J. C. \& Bernstein, I. H. (1994). Psychometric theory ( $3^{\text {rd }}$ ed.). New York, NY: McGraw-Hill 
Pallant, J. (2013). SPSS survival manual: A step by step guide to data analysis using IBM statistics $\left(5^{\text {th }}\right.$ ed.). New York, NY: McGraw Hill.

Pirgon, Ö., Sandal, G., Gökçen, C., Bilgin, H., \& Dündar, B. (2015). Social anxiety, depression, and self-esteem in obese adolescent girls with acanthosis nigricans. Journal of Clinical Research in Pediatric Endocrinology, 7, 63-68. doi: $10.4274 /$ jcrpe. 1515

Polit, D. F. \& Beck, C. T. (2012). Nursing research: Generating and assessing evidence for nursing practice (9th ed.). Philadelphia, PA: Wolters Kluwer Health | Lippincott, William, \& Wilkins.

Pollock, C. M., Harries, R. L., Smith, A. J., Straker, L. M., Kendall, G. E., \& O'Sullivan, P. B. (2011). Neck/shoulder pain is more strongly related to depressed mood in adolescent girls than boys. Manual Therapy, 16, 246-251.

doi:10.1016/jmath.2010.10.010

Richardson, L. P., McCauley, E., Grossman, D. C., McCarty, C. A., Richards, J., Russo, J. E., ...Katon, W. (2010). Evaluation of the Patient Health Questionnaire (PHQ9) for detecting major depression among adolescents. Pediatrics, 126, 1117-1123. doi:10.1542/peds.2010-0852

Rofey, D. L., Szigethy, E. M., Noll, R. B., Dahl, R. E., Lobst, E., \& Arslanian, S. A. (2009). Cognitive-behavioral therapy for physical and emotional disturbances in adolescents with polycystic ovary syndrome: A pilot study. Journal of Pediatric Psychology, 34, 156-163. doi:10.1093/jpepsy/jsn057

Romo, A. \& Benavides, S. (2008). Treatment options in insulin resistance obesity-related acanthosis nigricans. Annals of Pharmacotherapy, 42, 1090-1094. doi:10.1345/aph.1K446

Rotterdam PCOS Consensus Group. (2004). Revised 2003 consensus on diagnostic criteria and long-term health risks related to polycystic ovary syndrome (PCOS). Human Reproduction, 19, 41-47. doi:10.1093/humrep/deh098

Saldaña, J. (2013). The coding manual for qualitative researchers $\left(2^{\text {nd }} \mathrm{ed}\right.$.). Thousand Oaks, CA: Sage.

Schmidt, P. J., Murphy, J. H., Haq, N., Danaceau, M. A., \& Simpson-St. Clair, L. (2002). Basal plasma hormone levels in depressed perimenopausal women. Psychoneuroendocrinology, 27, 907-920. doi:10.1016/S0306-4530(02)00004-5 
Schuiling, K. D. \& Low, L. K. (2006). Women's growth and development across the life span. In K. D. Schuiling \& F. E. Likis (Eds.), Women's gynecologic health (pp. 21-39). Sudbury, MA: Jones and Bartlett.

Seiffge-Krenke, I. (1995). Stress, coping, and relationships in adolescence. Hillsdale, NJ: Lawrence Erlbaum.

Sequela. (2015). In Merriam-Webster's online dictionary. Retrieved from http://www.merriam-webster.com/dictionary/sequela

Streubert, H. J. \& Carpenter, D. R. (2011). Qualitative research in nursing: Advancing the humanistic imperative. Philadelphia, PA: Lippincott Williams \& Wilkins.

Thomsen, A. H., Compas, B. E., Colletti, R. B., Stanger, C., Boyer, M. C., \& Konik, B. S. (2002). Parents reports of coping and stress responses in children with recurrent abdominal pain. Journal of Pediatric Psychology, 27, 215-226.

doi:10.1093/jpepsy/27.3.215

Townsend, C. O. (2002). Perceived stress, coping and dietary fat intake in rural adolescents: gender and ethnic differences. Dissertation Abstracts International: Section B: The Sciences and Engineering, 63, 1052.

Trent, M. E., Austin, S. B., Rich, M., \& Gordon, C. M. (2005). Overweight status of adolescent girls with polycystic ovary syndrome: Body mass index as mediator of quality of life. Ambulatory Pediatrics, 5, 107-111. doi:10.1367/A04-130R.1

Trent, M. E., Rich, M., Austin, S. B., \& Gordon, C. M. (2002). Quality of life in adolescent girls with polycystic ovary syndrome. Archives of Pediatrics \& Adolescent Medicine, 156, 556-560.

University of Miami. (2007). Brief COPE. Retrieved from http://www.psy.miami.edu/faculty/ccarver/sclBrCOPE.html

Usmiani, S. \& Daniluk, J. (1997). Mothers and their adolescent daughters: Relationship between self-esteem, gender role identity, and body image. Journal of Youth and Adolescence, 26, 45-62.

Weber, B., Lewicka, S., Deuschle, M., Colla, M., \& Heuser, I. (2000). Testosterone, androstenedione, and dihydrotestosterone concentrations are elevated in female patients with major depression. Psychoneuroendocrinology, 25, 765-771. doi:10.1016/S0306-4530(00)00023-8 
Weiner, C. L., Primeau, M., \& Ehrmann, D. A. (2004). Androgens and mood dysfunction in women: Comparison of women with polycystic ovarian syndrome to healthy controls. Psychosomatic Medicine, 66, 356-362. Retrieved from http://www.ncbi.nlm.nih.gov/pubmed/15184695

Weiss, T. R. \& Bulmer, S. M. (2011). Young women's experiences living with polycystic ovary syndrome. Journal of Obstetric, Gynecologic, \& Neonatal Nursing, 40, 709-718. doi:10.1111/j.1552-6909.2011.01299.x

Wiebe, D. J., Berg, C.A., Korbel, C., Palmer, D. L., Beveridge, R. M., Upchurch, R., ,.. Donaldson, D. L. (2005). Children's appraisals of maternal involvement in coping with diabetes: Enhancing our understanding of adherence, metabolic control, and quality of life across adolescence. Journal of Pediatric Psychology, 30, 167-178. doi:10.1093/jpepsy/jsi004

Wild, R. A., Painter, P. C., Coulson, P. B., Carruth, K. B., \& Ranney, G. B. (1985). Lipoprotein lipid concentrations and cardiovascular risk in women with polycystic ovary syndrome. Journal of Clinical Endocrinology and Metabolism, 61, 946-951. Retrieved from http://www.ncbi.nlm.nih.gov/pubmed/4044782

Wilson, G. S., Pritchard, M. E., \& Revalee, B. (2005). Individual differences in adolescent health symptoms: The effects of gender and coping. Journal of Adolescence, 28, 369-379. doi:10.1016/j.adolescence.2004.08.004

Youngster, M., Ward, V. L., Blood, E. A., Barnewolt, C. E., Emans, S. J., \& Divasta, A. D. (2014). Utility of ultrasound in the diagnosis of polycystic ovary syndrome in adolescents. Fertility and Sterility, 102, 1432-1438.

doi:10.1016/j.fertnstert.2014.07.1241

Zeidner, M. \& Saklofske, D. (1996). Adaptive and maladaptive coping. In M. Zeidner \& N. S. Endler (Eds.), Handbook of coping: Theory, research, applications (pp. 505-531). New York, NY: John Wiley \& Sons, Inc.

Zimmer-Gembeck, M. J. \& Skinner, E. A. (2015). Adolescent vulnerability and the distress of rejection: Associations of adjustment problems and gender with control, emotions, and coping. Journal of Adolescence, 45, 149-159. doi:10.1016/j.adolescence.2015.09.004 
APPENDICES 
APPENDIX A

DEMOGRAPHIC FORM 


\section{Demographic Form}

1. What is your age in years?

2. Have you been diagnosed with polycystic ovary syndrome (PCOS)?

\begin{tabular}{c} 
Yes \\
\hline No
\end{tabular}

3. If yes, at what age were you when you were diagnosed with PCOS?

4. Circle symptoms you have experienced or have been diagnosed with:

Irregular periods Abnormal or extra hair growth (hirsutism) High testosterone

5. What is your race?

White, non-Hispanic

Hispanic

Black

Asian/Pacific Islander

Multiracial

Other

6. What is your ethnicity?

Non-Hispanic/Non-Latina

Hispanic/Latina

7. What type of health insurance do you have?

Medicaid

Private insurance

Uninsured

I don't know 
APPENDIX B

PCOS STRESSOR SCALE 
These Itoms deal with the most common issues/concerns giris and women with PCOS expertence. Answer oach item by circling the cholce that best reflects your thoughts and feelings about PCOS issues/concerns. For the ltems about your mother. this can be your blological mother, adoptive mother, or anyone you consider to be your mother-figure.

Respond to the following issues based on how concerned you an abour them

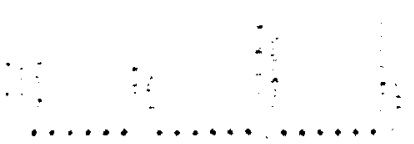

$A: \therefore$

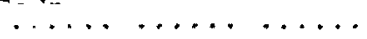

$41,4115 x$

\& $\cdots,+, \ldots, \cdots, \ldots, \ldots$

$\ldots \ldots \ldots \ldots$

VEE AT GOEE?

$\ldots \ldots \ldots \ldots . . .$.

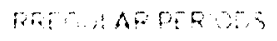

$\ldots \ldots \ldots \ldots$

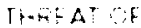

GHAN NGSASE

$\ldots \ldots \ldots$

THEAT CH

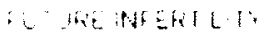

, ... .........
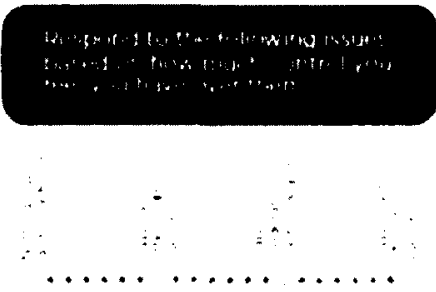

$A C N=$

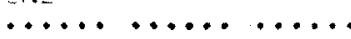

iffot: sm

$4 \ldots+\ldots \ldots$
$\ldots \ldots$$\ldots \ldots$

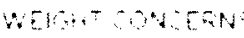

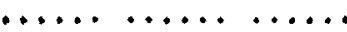

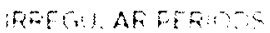

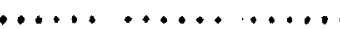

HRTAT G

HAOC: - TSASE

$\ldots \ldots \ldots \ldots \ldots$

TARAT $\rightarrow 5$

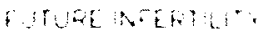

$\ldots \ldots \ldots \ldots \ldots$
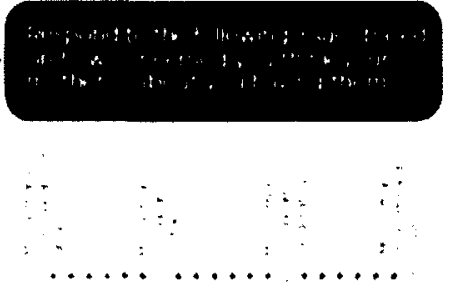

$A i_{2} \cdot v$

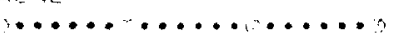

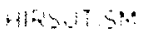

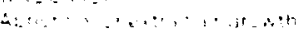

$\ldots \ldots \ldots \ldots \ldots \ldots . . . \ldots$

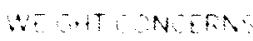

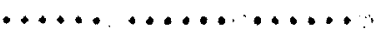

WRE ISARPTRLT:

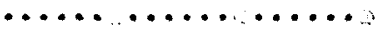

HAFA

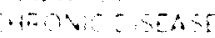

$\ldots \ldots \ldots \ldots$

$3+105 a^{2}$

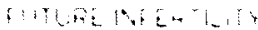

............. 
APPENDIX C

BRIEF COPE 


\section{Brief COPE}

These items deal with ways you've been coping with the stress in your life since you found out you had polycystic ovary syndrome (PCOS) or were having signs and symptoms such as irregular periods, abnormal or extra hair growth, high testosterone, weight problems, or acne.

There are many ways to try to deal with problems. These items ask what you've been doing to cope with this one. Obviously, different people deal with things in different ways, but I'm interested in how you've tried to deal with it. Each item says something about a particular way of coping. I want to know to what extent you've been doing what the item says. How much or how frequently. Don't answer on the basis of whether it seems to be working or not-just whether or not you're doing it.

Use these response choices below; write your answer $(1,2,3$, or 4$)$ in the space provided after each item. Try to rate each item separately in your mind from the others. Make your answers as true FOR YOU as you can.

$$
\begin{aligned}
& 1=I \text { haven't been doing this at all } \\
& 2=\text { I've been doing this a little bit } \\
& 3=\text { I've been doing this a medium amount } \\
& 4=\text { I've been doing this a lot }
\end{aligned}
$$

1. I've been turning to school, work, or other activities to take my mind off things.

2. I've been concentrating my efforts on doing something about the situation I'm in.

3. I've been saying to myself "this isn't real."

4. I've been using alcohol or other drugs to make myself feel better.

5. I've been getting emotional support from others.

6. I've been giving up trying to deal with it.

7. I've been taking action to try to make the situation better.

8. I've been refusing to believe that it has happened.

9. I've been saying things to let my unpleasant feelings escape.

10. l've been getting help and advice from other people.

11. I've been using alcohol or other drugs to help me get through it.

12. I've been trying to see it in a different light, to make it seem more positive.

13. I've been criticizing myself.

14. l've been trying to come up with a strategy about what to do.

15. I've been getting comfort and understanding from someone.

16. I've been giving up the attempt to cope.

17. l've been looking for something good in what is happening.

18. l've been making jokes about it.

19. l've been doing something to think about it less, such as going to movies, watching TV, reading, daydreaming, sleeping, or shopping.

20. I've been accepting the reality of the fact that it has happened. 
21. I've been expressing my negative feelings.

22. I've been trying to find comfort in my religion or spiritual beliefs.

23. I've been trying to get advice or help from other people about what to do.

24. I've been learning to live with it.

25. I've been thinking hard about what steps to take.

26. I've been blaming myself for things that happened.

27. I've been praying or meditating.

28. I've been making fun of the situation. 
APPENDIX D

PATIENT HEALTH QUESTIONNAIRE (PHQ-9) 
PATIENT HEALTH QUESTIONNAIRE (PHQ-9)

NAME

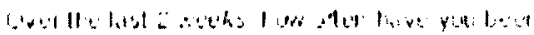

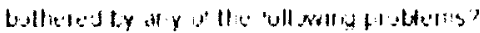

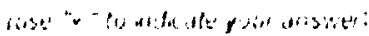

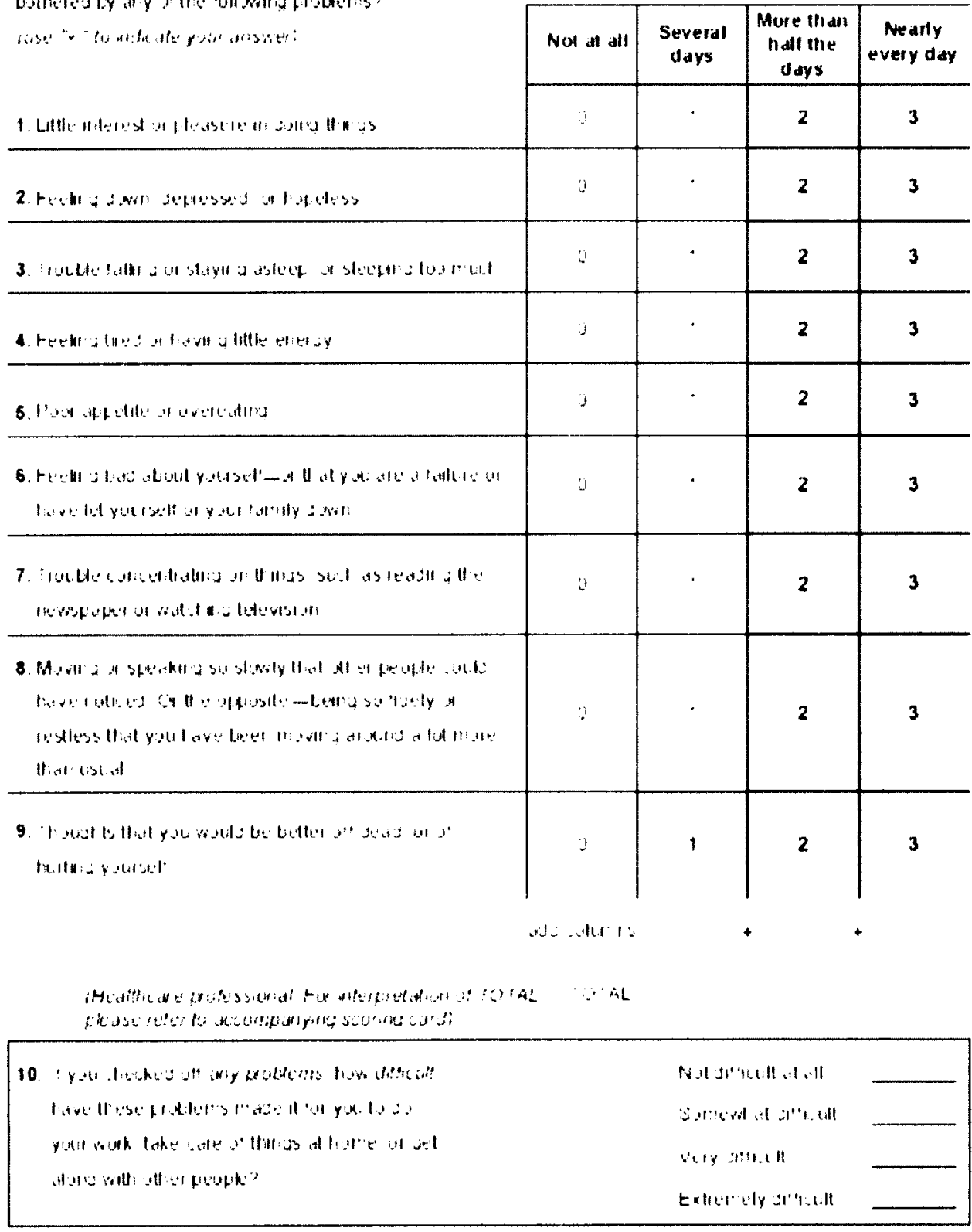

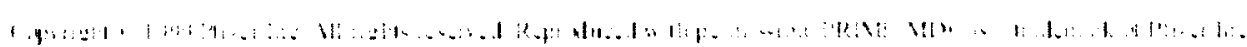
i2, +.

DATE 


\section{PHQ-9 Patıent Depression Questionnaire}

For initial diagnosis:

1 Panent completes PHQ-9 Qunck Depression A ssessment

2 If there are at least $4 \gamma_{5}$ un the shaded section (uncludug Questions 1 and $\cdots$ ? consider a depressive disorder Add score to determine setern:

Corrsider Major Dopressive Disorder

- If there are at least $5 \checkmark_{s}$ in the shaded sectuon 'ove of which conesponds to Questaon 1 or $=$ ?:

Cousider Other Depressive Disorder

- If there are 2.t $r$ s in the shaded secton ione of wheb conesponds to Question 11 or "I.

Yore: Sunce the quesnonnaure selies on panent self-report. all responses should be vernied tov the ciuncian. and a definubie diagnosis is made on clincal grounds taling into arcount how well the patent understood the questonnare. as well as other releimt unfomation from the patent

Diagnoses of Major Depressive Disorder or Other Depressive Disorder aiso require umparment of ivial. occupational or other unportant areas of functionug i Question 10 , and nuling out nomal bereavement. a bustony of a Manic Episode (Bupolar Disorder). and a physical disorder medicanon or other drug as the biological cause of the depressive simptoms

To monitor sereriti orer nime for aenly diagnosed patieats or parients in current rre atment fot deptession:

1 Patuents may complete questionnares at baselme and at regular unterials leg. eien: a week: 1 at home and bing them in at their next appointment for sconing or ther may complete the questomaure durng each scbectuled appoutroent

2 Add up $\checkmark s$ by column For every $\checkmark$ Several days $=1$. Wore than balf the days $=2$ Nearly e:er day $=$ :

3 Add rogethes colum scores to get 2 TOTAL score

+ Refer to the accompaning PHQ-9 Scoring Box to interpret the TOTAL score

5 Results my be uncluded in panent fles to assist you in serong up a treatment goal. determung degree of response. as well as gunding treatment interiention

Scoring: add op all checked boxes on PHQ-9

For every $\checkmark$ Not at all $=0$ Secieral days $=1$

She than half the days $=$ ?. Nearly eiery day $=$;

Interpretation of Toral Score

\begin{tabular}{|c|c|}
\hline Toed Scare & Depressien Severiti \\
\hline $1-1$ & Minimal depression \\
\hline 5.9 & Weld depression \\
\hline 10.14 & Moderate depression \\
\hline $15-19$ & Moderately seviete depression \\
\hline $30.3^{n}$ & Seriere depression \\
\hline
\end{tabular}

PHQ9 Copinght 5 Pfizer Inc All nghts resened Peproduced with permission PPNIE-AD \& is a tademark of Pinzer Inc

A.66:B 10.01.2005 
APPENDIX E

INTERVIEW GUIDE 
Interview Guide

Grand Tour Question: What is it like to have PCOS or to deal with symptoms such as irregular periods, hirsutism, acne, weight problems?

1. What is the most stressful thing about having PCOS or dealing with the symptoms I have described?

2. Describe your sense of control over the symptoms you experience related to PCOS or the symptoms you experience?

3. Describe your sense of control over potential negative health implications related to PCOS or the symptoms you experience?

4. What things do you do to cope with having PCOS and/or the related symptoms?

5. How has PCOS or the symptoms you experience affected your relationship with your family and friends?

6. Is there anything else you would like to tell me? 
APPENDIX F

INSTITUTIONAL REVIEW BOARD APPROVAL 
$22-0 a-2015$

Ms Casey 5 . Mopkins

ceorgin Beptist school of Mursing

Admunistriotion

1400 colemon svenue

Macon, GA 31207-0001

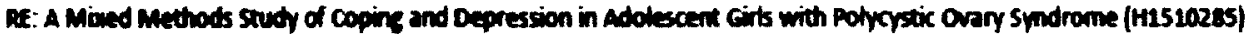

Dear Ms. Mopkins

Your applixation entitled A Mised Methods study of Coping and Depression in Adolescent Gris with Potycystx Over Syndrome (H1510255) wrs

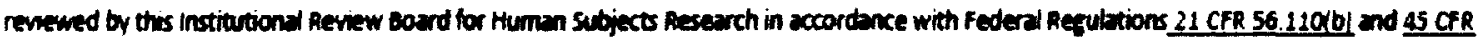
\$6.11901bilfor expedted review/ and was approved under category 7 per 63 fR 60364

Your applicacon was approved for one vear of study on 22-0ct-2015. The protocol expires 21-oct-2016. If the study continues beyond one year, it must be re-evaluated by the IRS committes.

trem(s) Approved:

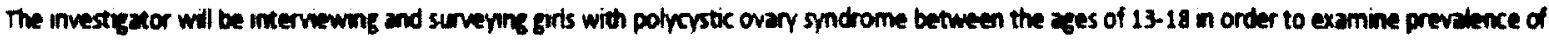
psychological discrders in this demogrephic, along with the ways gris cope with PCOS. Use of audo recording. Approved with a wainer of HipaA arthonzation as healthcare providers will dentify didive participants and give them the research padet pertiopants will then contect the investegator should they went to participate in the study. At thet bime ther will sign the consent form for partioption in the study

Please complete the survey for the lRB and the Office of Research compliance to access the surver, ctich on the following

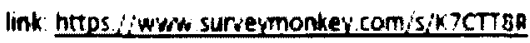

nespectiuly.

$$
\therefore \text {....... x...... }
$$

Ava Chambliss-Richardson, MED., CIP, OM

Member

Intutional Review board

Merce University IRo \& Office of Research compliance

Phone (478) 301-4101

Fax [478) $301-2329$

OAC Merceremercer Edu 